\title{
Developmental outcomes of early adverse care on amygdala functional connectivity in nonhuman primates
}

\author{
Elyse L. Morin ${ }^{1,2}$, Brittany R. Howell ${ }^{1,2,3,4}$, Eric Feczko ${ }^{5}$, Eric Earl ${ }^{5}$, Melanie Pincus ${ }^{1,2}$, Katherine Reding ${ }^{1}$, \\ Zsofia A. Kovacs-Balint ${ }^{2}$, Jerrold S. Meyer ${ }^{6}$, Martin Styner ${ }^{7}$, Damien Fair ${ }^{5}$ and Mar M. Sanchez ${ }^{1,2}$ \\ ${ }^{1}$ Yerkes National Primate Research Center, Emory University, Atlanta, GA, USA; ${ }^{2}$ Department of Psychiatry \& Behavioral Sciences, Emory University, Atlanta, GA, \\ USA; ${ }^{3}$ Fralin Biomedical Research Institute at VTC, Roanoke, VA, USA; ${ }^{4}$ Department of Human Development and Family Science, Virginia Tech, Blacksburg, VA, \\ USA; ${ }^{5}$ Department of Behavioral Neuroscience, Oregon Health and Science University, Portland, OR, USA; ${ }^{6}$ Department of Psychological and Brain Sciences, \\ University of Massachusetts, Amherst, MA, USA and ${ }^{7}$ Departments of Psychiatry and Computer Science, University of North Carolina, Chapel Hill, NC, USA
}

\begin{abstract}
Despite the strong link between childhood maltreatment and psychopathology, the underlying neurodevelopmental mechanisms are poorly understood and difficult to disentangle from heritable and prenatal factors. This study used a translational macaque model of infant maltreatment in which the adverse experience occurs in the first months of life, during intense maturation of amygdala circuits important for stress and emotional regulation. Thus, we examined the developmental impact of maltreatment on amygdala functional connectivity (FC) longitudinally, from infancy through the juvenile period. Using resting state functional magnetic resonance imaging (MRI) we performed amygdala-prefrontal cortex (PFC) region-of-interest and exploratory whole-brain amygdala FC analyses. The latter showed (a) developmental increases in amygdala FC with many regions, likely supporting increased processing of socioemotional-relevant stimuli with age; and (b) maltreatment effects on amygdala coupling with arousal and stress brain regions (locus coeruleus, laterodorsal tegmental area) that emerged with age. Maltreated juveniles showed weaker FC than controls, which was negatively associated with infant hair cortisol concentrations. Findings from the region-of-interest analysis also showed weaker amygdala FC with PFC regions in maltreated animals than controls since infancy, whereas bilateral amygdala FC was stronger in maltreated animals. These effects on amygdala FC development may underlie the poor behavioral outcomes associated with this adverse experience.
\end{abstract}

Keywords: amygdala functional connectivity, childhood maltreatment, early life stress, prefrontal cortex, rhesus monkey

(Received 31 January 2020; revised 16 May 2020; accepted 19 May 2020)

\section{Introduction}

Childhood maltreatment, including neglect, and physical, emotional, or sexual abuse, is a major public health concern experienced by approximately 1 in 4 US children (Finkelhor, Turner, Shattuck, \& Hamby, 2013). Maltreatment is a devastating form of early life stress (ELS) and is associated with increased risk for anxiety and depression, posttraumatic stress disorder (PTSD), cognitive dysfunction, behavioral disorders, substance abuse, obesity, and inflammation, in both humans and nonhuman primates (NHPs) (Asok, Bernard, Roth, Rosen, \& Dozier 2013; Danese \& Tan, 2014; Drury et al., 2012; Drury, Sanchez, \& Gonzalez, 2016; Gee et al., 2013; Gunnar \& Quevedo, 2007; Howell \& Sanchez, 2011; McLaughlin et al., 2015; Petrullo, Mandalaywala, Parker, Maestripieri, \& Higham, 2016; Rutter et al., 1999; Sanchez et al., 2007; Teicher et al., 2003). However, the developmental consequences of maltreatment vary in complex ways depending on several factors, including the duration and age of exposure (Kaplow \& Widom, 2007; Kisiel et al.,

Author for Correspondence: Dr. Mar Sanchez, Department of Psychiatry and Behavioral Sciences, and Yerkes National Primate Research Center, Emory University, 954 Gatewood Drive NE, NS 4216, Atlanta, GA 30329; E-mail: mmsanch@emory.edu.

Cite this article: Morin EL et al (2020). Developmental outcomes of early adverse care on amygdala functional connectivity in nonhuman primates. Development and Psychopathology 32, 1579-1596. https://doi.org/10.1017/S0954579420001133
2014; Spinazzola et al., 2014; Steinberg et al., 2014), form and severity of maltreatment (i.e., physical abuse, neglect, sexual abuse -which are often co-morbid-), and co-occurrence with other forms of emotional and psychological trauma (Kisiel et al., 2014; Spinazzola et al., 2014). Despite the well-established links between early life adversity and the emergence of psychopathology, the specific neurobiological and developmental mechanisms that translate early adversity into poor health outcomes are not well understood, due in part to the complexities described above.

Alterations in the development of brain networks that control arousal, stress, threat, and emotional responses, particularly the amygdala and its connectivity with prefrontal cortex (PFC), have been proposed as potential neural mediators of the behavioral alterations associated with ELS (Foa \& Kozak, 1986; Teicher, Samson, Anderson, \& Ohashi, 2016; VanTieghem \& Tottenham, 2018; Weber, 2008). However, understanding how these neurobiological changes unfold has been challenging. This stems in part from the challenges inherent in conducting prospective, longitudinal studies in children at risk, lack of experimental control, and complex comorbid conditions and environmental confounds (e.g., diet, prenatal exposure to drugs or alcohol, access to health care, socioeconomic status - SES -, etc.). As an alternative, rhesus monkeys (Macaca mulatta) provide an ethologically valid, translational animal model to address the questions raised 
in human studies through longitudinal, prospective experimental designs that can combine random assignment of infant monkeys to rearing groups at birth with collection of highly dense, longitudinal neurobehavioral sampling from infancy through the juvenile period. Additional strengths of the NHP model for examining the impact of maternal maltreatment include the ability to apply longitudinal magnetic resonance imaging (MRI) techniques, neurodevelopmental speed that progresses about four times faster than humans, and complex social behavior, including motherinfant attachment (Howell \& Sanchez, 2011; Sanchez, 2006; Sanchez, Ladd, \& Plotsky, 2001).

The quality of the relationship between a macaque mother and her infant is, as in humans, highly influential of offspring neurobehavioral development (Hinde \& Spencer-Booth, 1967). Poor maternal care in the form of infant maltreatment, including abuse and rejection, occurs in NHP species spontaneously and with prevalence rates similar to humans' (2\%-5\%) (Brent, Koban, \& Ramirez, 2002; Johnson, Kamilaris, Calogero, Gold, \& Chrousos, 1996; Maestripieri \& Carroll, 1998; Sanchez, 2006; Troisi \& D’Amato, 1984). Developmental consequences include chronic activation of stress response systems and alterations in socioemotional functioning (Bramlett et al., 2017, 2018; Brent et al., 2002; Drury et al., 2017; Howell, Neigh, \& Sánchez, 2016; Johnson et al., 1996; Maestripieri \& Carroll, 1998; Morin, Howell, Meyer, \& Sanchez, 2019; Sanchez, 2006; Sanchez, Hearn, Do, Rilling, \& Herndon, 1998; Troisi \& D’Amato, 1984). The naturalistic rhesus model of maternal infant maltreatment studied here is operationalized as both early life physical abuse and comorbid maternal rejection, both of which are associated with infant distress (Maestripieri, 1999; Maestripieri \& Carroll, 1998; McCormack et al., 2009; McCormack, Sanchez, Bardi, \& Maestripieri, 2006; Sanchez, 2006). As in humans, there is transgenerational transmission of maltreatment through the maternal line in macaques (Maestripieri, 2005; Maestripieri \& Carroll, 1998). Maltreating mothers reliably maltreat subsequent offspring, suggesting this is a stable maternal trait (Maestripieri, 2005; Maestripieri \& Carroll, 1998). Such generational perpetuation of maltreatment parallels findings in humans with a history of childhood adverse care, providing further validation of this NHP model for use in studies of mechanisms underlying developmental outcomes (Franklin et al., 2010; Huizinga et al., 2006; Maestripieri, 2005; Tarullo \& Gunnar, 2006).

Macaque infant studies have recapitulated alterations reported in maltreated children (and those with other adverse caregiving experiences), including increased anxiety, emotional reactivity and aggression, impaired impulse control, social deficits, elevated levels of stress hormones indicative of chronic stress exposure, activation of pro-inflammatory pathways, structural alterations in cortico-limbic tracts, and larger amygdala volumes (Bramlett et al., 2017, 2018; Drury et al., 2017; Howell et al., 2019; Howell et al., 2014; Howell et al., 2013; Koch, McCormack, Sanchez, \& Maestripieri, 2014; Maestripieri, 1998; McCormack, Newman, Higley, Maestripieri, \& Sanchez, 2009; McCormack et al., 2006; Morin et al., 2019; Petrullo et al., 2016; Sanchez, 2006; Sanchez et al., 2010). Of particular interest for this study are the maltreatment effects on the structural integrity of cortico-limbic white matter tracts involved in socioemotional processing and responses during rhesus development previously reported by our group (Howell et al., 2013; Howell et al., 2017; Howell et al., 2019). It is possible that repeated exposure to elevated levels of cortisol due to the adverse experience, among other factors, alters the microstructural organization of white matter tracts through alterations in cellular developmental processes that contribute to these indices of microstructural organization, including myelination, leading to subsequent impacts in functional connectivity (FC) between these regions.

Myelination of axons is a critical cellular process that serves to increase conductance of action potentials, supporting FC between distant brain regions (Fields, 2008; Thomason \& Thompson, 2011; Zatorre, Fields, \& Johansen-Berg, 2012). Myelination changes drastically during early development (Deoni, Dean, O’Muircheartaigh, Dirks, \& Jerskey, 2012; Dubois et al., 2014; Eluvathingal et al., 2006; Geng et al., 2012; Govindan, Behen, Helder, Makki, \& Chugani, 2010; Kumar et al., 2014). This rapid increase in myelination early in life makes this process vulnerable to environmental influences, including adverse care and ELS, and the elevated glucocorticoid levels associated with these experiences (Jauregui-Huerta et al., 2010; Kumar, Cole, Chiappelli, \& de Vellis, 1989). In addition, suboptimal maternal care also impacts the ability of the mother to buffer the infant's stress, fear, and arousal responses (Sanchez, McCormack, \& Howell, 2015). These infant's responses are buffered through maternal signals that regulate emotion and stress circuitry (Howell et al., 2017), especially amygdala connections with the hypothalamus and relevant brain stem regions, as well as with PFC, for top-down emotional regulation (Moriceau \& Sullivan, 2006). Thus, maternal care seems to play a critical role in the development of these self-regulatory amygdala circuits as infants reach independence (Drury et al., 2016; Gee et al., 2014; Gunnar \& Sullivan, 2017; Gunnar, Hostinar, Sanchez, Tottenham, \& Sullivan, 2015; Sanchez et al., 2015; Tottenham, 2015), providing a likely mechanism through which infant maltreatment impacts neural and socioemotional development (Casey, Duhoux, \& Malter Cohen, 2010; Fairbanks, 1996; Maestripieri \& Carroll, 1998). As recently reviewed by VanTieghem and Tottenham (VanTieghem \& Tottenham, 2018), although different forms of early adversity in children impact amygdala-prefrontal circuits, issues related to the chronicity and complexity of these experiences in humans do not allow differentiation of timing (age) versus duration effects. Thus, the authors called for preclinical studies with animal models of ELS to address these questions using longitudinal designs.

Thus, in this study we used a translational NHP model of infant maltreatment that occurs during an early and discrete developmental phase (the first 3-6 months of life, equivalent to the infant and toddlerhood period in humans) to examine: (a) its longitudinal impact on development of amygdala FC, which may underlie the increased emotional reactivity reported in maltreated animals (Howell et al., 2014; McCormack et al., 2009; McCormack et al., 2006; Morin et al., 2019; Morin et al., 2018); and (b) whether the prolonged exposure to high cortisol during infancy reported in maltreated animals - measured as high hair cortisol concentrations from birth through 6 months (Drury et al., 2017) - predicted alterations in amygdala FC. Although this NHP model of infant maltreatment does not span all adversity that maltreated children experience (e.g., sexual abuse), one of its critical strengths lies in its ability to quantify maltreatment during a known postnatal period, providing frequency, duration, and severity of the adverse experience (i.e., abuse and rejection rates), as well as the concurrent levels of stress it elicits (i.e., cortisol accumulation in hair during the postnatal ELS exposure), all of which are difficult to measure in children. NHP models also provide strong control over environmental variables that are known confounders of behavioral outcomes of ELS in human studies, such as drug use, nutrition, obesity, prenatal stress, SES, 
and access to medical care. Our experimental design also provides a tool to control for the effects of heritable and prenatal factors on postnatal experience by utilizing cross-fostering and randomized assignment to experimental group at birth (Drury et al., 2017; Howell et al., 2017; Howell et al., 2019), which is not possible in humans.

Thus, we used this translational NHP model of infant maltreatment to examine developmental alterations in amygdala FC longitudinally, from infancy through the juvenile prepubertal period (at 3, 6, 12, 18 months of age) which could underlie behavioral and stress outcomes already reported in these animals (Drury et al., 2017; Howell et al., 2017; Howell et al., 2019). We collected resting state functional MRI (rsfMRI) data to examine the developmental impact of maltreatment on amygdala FC (a) with the whole brain, using an exploratory voxel-wise amygdala seed-based FC analysis at the group level; in parallel to (b) specific region of interest (ROI) analysis to examine alterations in amygdala-PFC FC. Based on previous work in both humans and NHPs we predicted that maltreated animals would show decreases in amygdala-PFC FC as they aged. In addition, we examined whether the elevated hair cortisol accumulation from birth through 6 months of age reported in maltreated infants (Drury et al., 2017) - a marker of chronic exposure to stress - predicted developmental changes in amygdala FC.

\section{Method}

\section{Subjects}

These studies included 20 rhesus macaques (Macaca mulatta) raised by their mothers in large social groups at the Yerkes National Primate Research Center Field Station (YNPRC, Lawrenceville, GA). Animals were studied from birth through to 18 months of age (juvenile, prepubertal age) as part of a larger longitudinal study of developmental consequences of infant maltreatment (Drury et al., 2017; Howell et al., 2017; Howell et al., 2019; McCormack et al., 2015; Morin et al., 2019). In this model, maternal infant maltreatment is a spontaneous and stable maternal trait exhibited during the first 3-6 months postpartum that leads to infant distress (Maestripieri, 1999; Maestripieri \& Carroll, 1998; Sanchez, 2006) and activation of infant stress systems (e.g., the hypothalamic-pituitary-adrenal (HPA) axis) (Bramlett et al., 2017, 2018; Drury et al., 2017; Howell et al., 2013). Half of the animals in this study experienced maternal maltreatment, including physical abuse and rejection (maltreated, $n=$ 10; 6 males, 4 females), and the other half received high quality maternal care (control, $n=10 ; 4$ males, 6 females). Subjects lived with their mothers and families in large social groups with a matrilineal social hierarchy consisting of 75-150 adult females, their subadult, juvenile and infant offspring, and 2-3 adult breeder males. Based on this social complexity we were able to use a balanced distribution of social dominance ranks, in addition to sex, across experimental caregiving groups. Infants were assigned from different matrilines and paternities to provide high genetic and social diversity, as previously reported (Drury et al., 2017; Howell et al., 2017; Howell et al., 2019; Morin et al., 2019). Because birth weight is a strong predictor of neurobehavioral development in humans and NHPs (Coe \& Shirtcliff, 2004; Vohr et al., 2000), we only studied infants with $\geq 450$ gm birth weight, which is a safe veterinary clinical cut off to rule out prematurity in rhesus monkeys. Furthermore, while 7 of the animals were raised by their biological mothers ( 4 maltreated: 3 males, 1 female; 3 control: 1 male, 2 females), the other 13 were randomly assigned at birth to be cross-fostered (CF) to either mothers with a history of nurturing maternal care or to maltreating foster mothers ( 7 control CF: 3 males, 4 females; 6 maltreated CF: 3 males, 3 females), in an effort to counter-balance the potential confounding effect of biological factors (e.g., heritability, prenatal confounders) on the effect of postnatal caregiving experience, following published protocols by our group (Drury et al., 2017; Howell et al., 2017; Howell et al., 2019; Morin et al., 2019) and previous methods (Maestripieri, 2005). Social groups were housed in outdoor compounds, with access to climatecontrolled indoor areas. As well as a standard, high fiber, and lowfat monkey chow diet (Purina Mills Int., Lab Diets, St. Louis, $\mathrm{MO}$ ), seasonal fruits and vegetables were provided twice daily, in addition to enrichment items. Water was available ad libitum. All procedures were in accordance with the Animal Welfare Act and the U.S. Department of Health and Human Services "Guide for the Care and Use of Laboratory Animals" and approved by the Emory Institutional Animal Care and Use Committee (IACUC).

\section{Behavioral characterization of maternal care}

The infant rhesus maltreatment model and the methods used for selection of potential mothers and behavioral characterization of competent maternal care (control care) versus infant maltreatment have been described in previous publications (Drury et al., 2017; Howell et al., 2017; Howell et al., 2019; Maestripieri, 1998; Maestripieri \& Carroll, 1998; McCormack et al., 2006; McCormack et al., 2009; Morin et al., 2019). Briefly, because maltreating mothers consistently maltreat subsequent offspring -suggesting this is a stable maternal trait (Maestripieri, 2005; Maestripieri \& Carroll, 1998) - we identified potential multiparous control and maltreating mothers for assignment to experimental group based on maternal care quality exhibited with prior offspring. Following birth (and cross-fostering where applicable) we performed focal observations of maternal care across the first three postnatal months to substantiate and quantify maltreatment (rates of abuse and rejection) and competent maternal care (cradling, grooming, etc.) towards biological or fostered infants. These were $30 \mathrm{~min}$ long focal observations of mother-infant interactions performed on separate days (5 days/ week during month 1, 2 days/week during month 2, and 1 day/ week during month 3) for a total of $16 \mathrm{hr} /$ mother-infant pair. This observation protocol is optimal to document early maternal care in this species, given that physical abuse rates are the highest during the first month and decline by the third month, after which it is rarely observed (Drury et al., 2017; Howell et al., 2017; Maestripieri \& Carroll, 1998; McCormack et al., 2006). Observations were performed between 7:00 and 11:00 a.m., when monkeys are most active. Behavioral observations were collected by experienced coders (interobserver reliability $>90 \%$ agreement, Cohen $k>0.8$ ). Competent maternal care is defined as speciestypical behaviors, such as nursing, cradling, grooming, ventral contact, and protection (retrieve from potential danger, restrain) of the infant. In contrast, maltreatment is aberrant, defined as the co-morbid occurrence of physical abuse (operationalized as violent behaviors directed towards the infant that cause pain and distress, including dragging, crushing, throwing, etc.) and early infant rejection (i.e., prevention of ventral contact and pushing the infant away during the first weeks of life). Both abuse and rejection cause high levels of infant distress (e.g., scream vocalizations, tantrums) and elevations in stress hormones (Bramlett et al., 2017, 2018; 
Drury et al., 2017; Howell et al., 2013; Maestripieri \& Carroll, 1998; McCormack et al., 2006; Sanchez, 2006). Control foster mothers in this study exhibited competent maternal care (e.g., high maternal sensitivity, infant protection, and responsivity) (McCormack et al., 2015) and did not exhibit maltreating behaviors (Drury et al., 2017; Howell et al., 2017).

\section{MRI scanning session}

MRI images were acquired longitudinally, at 3 and 6 months (infancy), and at 12 and 18 months (juvenile period) of age using a 3T Siemens Magnetom Tim Trio MRI scanner (Siemens Med. Sol., Malvern, PA, USA), and an eight-channel phase array knee coil (YNPRC Imaging Center). Subjects were transported with their mothers from the YNPRC Field Station to the YNPRC Main Station on the main Emory campus in Atlanta on the day before or early in the morning of the day of the scan. All MRI data were acquired during a single scanning session, with the animals scanned supine in the same orientation and the head immobilized in a custom-made head holder with ear bars and a mouthpiece to minimize motion. A vitamin E capsule was placed on the right temple to mark the right side of the brain.

Following initial induction of light anesthesia with telazol (3.9 $\pm 0.83 \mathrm{mg} / \mathrm{kg} \mathrm{BW}$, i.m. mean \pm standard deviation) and intubation, scans were collected under the lowest possible level of isoflurane anesthesia $(1.0 \pm 0.1 \%$, inhalation; mean \pm standard deviation) to minimize its reported dampening effect on blood oxygen level dependent (BOLD signal) (Hutchison, Womelsdorf, Gati, Everling, \& Menon, 2013; Li, Patel, Auerbach, \& Zhang, 2013; Miranda-Dominguez et al., 2014b; Tang \& Ramani, 2016; Vincent et al., 2007). MRI images were acquired and processed following approaches optimized by our group for studies of macaque neurodevelopment (Kovacs-Balint et al., 2018; Mavigner et al., 2018) and protocols developed and widely used for macaques (Grayson et al., 2016; Hutchison et al., 2012; Li et al., 2013; Margulies et al., 2009; Sallet et al., 2011; Vincent et al., 2007). Our isoflurane levels are even lower than previously used in macaque studies of sensory, motor, visual, and cognitive-task related systems that reported patterns of coherent BOLD fluctuations similar to those observed in awake behaving monkeys (Vincent et al., 2007; Hutchison et al., 2013; Li et al., 2013; Miranda-Dominguez et al., 2014b; Tang \& Ramani, 2016). Ideally, these animals would have been scanned awake, but training socially housed infants and juveniles for awake scanning is not currently feasible. Each animal was fitted with an oximeter, rectal thermometer, and blood pressure and heart rate monitor for physiological monitoring during the scans. An intravenous catheter was placed in the saphenous vein to administer dextrose/ $\mathrm{NaCl}(0.45 \%)$ to maintain hydration. Each animal was placed on an MRI-compatible heating pad to maintain normal body temperature and was monitored by veterinary staff throughout the scan procedures. Infants were immediately returned to their mothers after full recovery from anesthesia, and mother-infant/ juvenile pairs were returned to their social groups the day after the scan.

\section{Structural MRI acquisition}

Structural images were acquired for registration of functional data to the age-specific atlas spaces. High-resolution structural MRI images (T1-weighted) were collected with a 3D magnetization prepared rapid gradient echo (3D-MPRAGE) parallel image sequence $(\mathrm{TR} / \mathrm{TE}=3000 / 3.31 \mathrm{~ms}, 116 \mathrm{~mm}$ FOV, voxel size: $0.6 \mathrm{~mm}^{3}, 6$ averages, GRAPPA, $\mathrm{R}=2$ ). T2-weighted scans were also collected in the same direction as the T1 during the same scanning session $(\mathrm{TR} / \mathrm{TE}=2500 / 84 \mathrm{~ms}, 128 \mathrm{~mm}$ FOV, voxel size: $0.7 \times 0.7 \times 2.0 \mathrm{~mm}, 1$ average, GRAPPA, $\mathrm{R}=2$ ) to aid with registration and delineation of anatomical borders.

\section{rsfMRI acquisition}

Two 15-min rsfMRI T2*-weighted scans with a gradient-echo echoplanar imaging (EPI) sequence $(400$ volumes, TR/TE $=$ 2060/25 msec, voxel size: $1.5 \mathrm{~mm}^{3}$ isotropic) were collected to measure temporal changes in regional BOLD signals. An additional short field map scan was also acquired for unwarping distortions in the EPI scans. The first three volumes were removed from each EPI scan to ensure scan environment stabilization, resulting in a total of 794 concatenated volumes per 15-min scan. These protocols and scan sequences have been optimized at the YNPRC for longitudinal infant macaque imaging (Kovacs-Balint et al., 2018; Mavigner et al., 2018; Shi et al., 2017; Zhang et al., 2017).

\section{rsfMRI data preprocessing}

Data pre-processing was done using the FMRIB Software Library (FSL, Oxford, UK; Smith et al., 2004; Woolrich et al., 2009), 4dfp, and an in-house pipeline built using Nipype (Gorgolewski et al., 2011), modified based on published functional MRI (fMRI) analysis methods (Fair et al., 2007; Fair et al., 2009; Fair et al., 2012; Iyer et al., 2013; Miranda-Dominguez et al., 2014a), and optimized for macaques (Miranda-Dominguez et al., 2014b), including resting state fMRI (rsfMRI) studies in infant rhesus by our group (Kovacs-Balint et al., 2018; Mavigner et al., 2018). This procedure consisted of (a) quantification and correction of dynamic field map changes, (b) slice-time correction of intensity differences as a result of interleaved slice image acquisition, (c) combined resampling of: within-run rigid-body motion correction, registration of the EPI to the subject's own averaged T1-weighted structural image, and registration of the $\mathrm{T} 1$ to age-specific T1-weighted rhesus infant and juvenile brain structural MRI atlases developed by our group (publicly available at: https://www.nitrc.org/projects/macaque_atlas), using nonlinear registration methods in FSL (FNIRT), (d) BOLD signal normalization to mode of 1000 , to scale BOLD values across participants at an acceptable range, (e) BOLD signal detrending, (f) regression of rigid body head motion (six directions), global brain signal, BOLD signal of the ventricles and white matter (derived from manually drawn masks), and first-order derivatives of these signals, and ( $g$ ) low-pass $(\mathrm{f}<0.1 \mathrm{~Hz}$ ) temporal filter (second order Butterworth filter) (Fair et al., 2007; Fair et al., 2009; Fair et al., 2012; Miranda-Dominguez et al., 2014b).

The infant and juvenile atlases were previously registered to the 112RM-SL atlas in F99 space (McLaren et al., 2009, 2010; Shi et al., 2017) as shown in (Miranda-Dominguez et al., $2014 \mathrm{~b}$ ) and were templates made using scans acquired longitudinally at 3, 6, and 12 months of age on 40 infant rhesus monkeys from the YNPRC social colony, balanced by sex and social rank. Based on best match of neuroanatomical characteristics, we registered the earliest scan age ( 3 months) to the 3 months atlas, the 6 months scans to the 6 months atlas and the later ages $(12,18$ months) to the 12 months rhesus atlas. All the atlases were transformed to conform to the T1-weighted 112RM-SL atlas 
image in F99 space, following previously described protocols (Miranda-Dominguez et al., 2014b) and allowing the EPI images to be transformed into F99 space in one interpolation step.

Global signal regression (GSR) was performed based on previous literature showing the importance of removing systematic artifacts, including global artifacts that arise as a consequence of movement, respiration, and other physiological noise (Burgess et al., 2016; Ciric et al., 2017; Nalci, Rao, \& Liu, 2017; Power, Laumann, Plitt, Martin, \& Petersen, 2017; Yan et al., 2013), including macaque rsfMRI FC studies (Grayson et al., 2016; Miranda-Dominguez et al., 2014b). Without doing so, spurious artifacts would become problematic, especially in longitudinal studies of FC throughout development. Notwithstanding the above defense of GSR, we acknowledge that controversy regarding this method persists (Murphy \& Fox, 2017). Because of this, we previously compared infant macaque FC with and without GSR and obtained similar results throughout development (KovacsBalint et al., 2018), including the current dataset, in which similar effects of maltreatment were detected on amygdala FC with and without GSR (Morin et al., 2015 ).

Motion artifacts were further minimized by removing frames displaced more than $0.2 \mathrm{~mm}$ (Power et al., 2014; Power, Barnes, Snyder, Schlaggar, \& Petersen, 2012). Following preprocessing, data quality was further inspected, and several scans were removed from the analysis due to a significant image acquisition artifact (nonbiological high intensity signal band passing axially through the temporal lobe). Therefore, of the 20 animals included in the study, 17 scans were included in the analyses at 3 months (2 were removed due to the image artifact, and 1 scan was not usable), all 20 scans were included at 6 months, 13 scans were included at 12 months ( 4 were removed due to image artifact, 1 scan was not usable and 2 animals could not be scanned due to sickness), and 8 scans at 18 months (1 scan was removed due to image artifact, 1 scan was not usable and the other 10 animals could not be scanned because they were no longer assigned to the study).

\section{Definition of amygdala and PFC ROIs}

ROIs were selected from published anatomical parcellations (Lewis \& Van Essen, 2000; Markov et al., 2011; Paxinos, Huang, \& Toga, 2000), defined using macaque atlases (Logothetis \& Saleem, 2012; Schmahmann \& Pandya, 2006), and mapped onto the 3, 6, and 12 months UNC (University of North Carolina)-Emory rhesus infant atlases (Shi et al., 2017) registered to the F99 space as described above. The amygdala ROI was drawn by neuroanatomists using cytoarchitectonic maps in the UNC-Wisconsin adolescent atlas (Styner et al., 2007), and propagated to the UNC-Emory rhesus 3, 6, and 12 months infant atlases in F99 space using deformable registration via ANTS (for details see Shi et al., 2017). The amygdala ROI included all amygdaloid nuclei, excluding perirhinal cortex. PFC ROIs were manually edited in each age-appropriate atlas according to established anatomical landmarks (Logothetis \& Saleem, 2012; Paxinos et al., 2000) and under guidance of an expert on amygdala and PFC developmental neuroanatomy (Jocelyne Bachevalier, Emory University), before removing voxels that covered regions where at least one animal showed signal dropout, determined as the mean intensity of each subjects' BOLD signal intensity across the whole-brain mask minus two standard deviations. PFC ROI subregions included the dorsolateral PFC (dlPFC) (Brodmann areas [BA] 9 \& 46), medial PFC (mPFC) (BA 25 \& 32), orbitofrontal cortex (OFC) (BA 11 \& 13), and anterior cingulate cortex (ACC) (BA 24).

\section{Whole brain voxel-wise amygdala FC analysis}

Seed-based maps of right and left amygdala FC with other voxels in the brain were created for each subject across all ages. A secondary seed-based analysis was limited to voxels which were significantly correlated with amygdala activity (false discovery rate (FDR)-corrected $q=0.05$, cluster size $\geq 10$ contiguous voxels), using data-reduction methods similar to those in previous macaque amygdala seed-based voxel-wise analyses (Grayson et al., 2016; Reding et al., 2020). These FC maps were included in a mixed linear model (MLM), AFNI's (Analysis of Functional NeuroImages) 3dLME (linear mixed-effects) statistical model, described in more detail in the Statistical Analysis section below. Statistically significant clusters after multiple comparisons correction using FDR were displayed on the infant atlases (Shi et al., 2017), and their anatomical localization identified with established combined stereotaxic/MRI rhesus macaque brain atlases (Logothetis \& Saleem, 2012; Paxinos et al., 2000); see Figure 2b.

\section{Amygdala-PFC FC: ROI-ROI analyses}

ROIs were selected, defined, and mapped to infant atlases as described above. BOLD time series were then parcellated and averaged across all voxels within each ROI, and averaged across the time course. Pearson correlations were calculated between amygdala and subregions of the PFC (dlPFC - BA 9, BA 46; mPFC -=- BA 25, BA 32; ACC - BA 24; OFC - BA 11, BA 13) for each age and Fisher Z-transformed to stabilize variance.

\section{Postnatal hair cortisol measures}

At birth, one square inch of hair was shaved from the infant's head just above the foramen magnum (nuccal area) in a subset of subjects (control: 3 male, 2 female; maltreated: 3 male, 2 female). Hair that had grown in that area was shaved again at 6 months of age. Hair samples collected at 6 months were assayed to measure cortisol accumulated in the growing hair shafts from birth through 6 months of age as a measure of HPA axis activity, which was higher in maltreated than control animals (Drury et al., 2017). Hair samples were processed and assayed using previously described protocols (Meyer, Novak, Hamel, \& Rosenberg, 2014). Hair was first weighed and washed in isopropanol to remove external contamination, then ground to powder and extracted with methanol overnight. After the methanol evaporated, the resulting residue was re-dissolved in assay buffer, and cortisol was measured using the Salimetrics (Carlsbad, CA) enzyme immunoassay kit (cat. \# 1-3002). Intraand inter-assay coefficients of variation were $<10 \%$.

\section{Statistical Analyses}

\section{Infancy-juvenile period longitudinal amygdala-PFC FC: ROI-ROI analyses}

Linear mixed models (LMM) were used to assess the effects of age $(3,6,12,18$ months), caregiving group (control, maltreated), sex (male, female), and hemisphere (right, left), on PFC-amygdala FC from infancy to the juvenile (prepubertal) period. The model was also run excluding sex for comparison with the amygdala-seed based voxel-wise LMM models. Post hoc 
comparisons were conducted using Student $t$ tests when significant caregiving group interaction effects were detected in the LMM, and Bonferroni corrected for multiple comparisons. Linear regression was used to test whether postnatal cortisol exposure was predictive of PFC-amygdala FC when significant caregiving group effects were detected in the LMM. Statistical significance level was set at $p<.05$.

\section{Longitudinal whole brain voxel-wise amygdala FC}

The seed-based maps of amygdala FC with other voxels in the brain were included in a MLM, AFNI's 3dLME group analysis program to examine the effects of age $(3,6,12,18$ months), caregiving group (control, maltreated) and hemisphere (right, left) on amygdala FC. Statistically significant voxel clusters following multiple comparison corrections using FDR (FDR-corrected $q=0.05$, cluster size $\geq 2$ voxels) are shown in Figure $2 b$. Sex was not included as a factor in these MLM models due to lack of power.

Post hoc Student $t$ tests were conducted on clusters with significant main group or group by age interaction effects, using Bonferroni correction for multiple comparisons. We then performed regression analyses to test whether early postnatal cortisol exposure were predictive of amygdala FC in clusters with significant group or group by age post hoc effects. FC in these clusters (Fisher's Z-transformed) was normally distributed. Statistical significance level was set at $p<.05$.

\section{Results}

\section{ROI-ROI FC}

\section{Left amygdala-right amygdala FC}

A significant main effect of group $\left(F_{(1,20.4)}=4.4, p=.0496\right)$ was detected with maltreated animals showing higher FC than controls across age (Figure 1a). Although a trend toward a main effect of age $\left(F_{(3,20.0)}=2.7, p=.0764\right)$ was found, no other significant main or interaction effects were detected.

When sex was excluded from the LMM model no main or interaction effects were detected.

\section{Amygdala-mPFC FC}

Amygdala-BA 25 FC. A significant group by sex interaction effect $\left(F_{(1,48.3)}=5.3, p=.0258\right)$ with higher FC in control than maltreated females was detected (Figure $1 \mathrm{~b}$ ); however, post hoc $t$ tests did not confirm significant differences between means. A group by sex by hemisphere interaction $\left(F_{(1,48.3)}=6.1, p=.0169\right)$ was also found, with higher positive FC in control than maltreated females in the right hemisphere $\left(t_{(1,12.0)}=2.3, p=.0431, \eta^{2}=\right.$ 0.187 ; Figure $1 b)$. No other significant main or interaction effects were detected.

When sex was excluded from the LMM model, no main or interaction effects were detected.

Amygdala-BA 32 FC. A significant main effect of age $\left(F_{(3,36.5)}=\right.$ 3.0, $p=.0426$ ) was detected, with the highest (positive) FC observed at 3 months, and decreasing through 18 months, although remaining positive (Figure 1a). A significant sex by hemisphere interaction effect was also found $\left(F_{(1,56.3)}=4.4\right.$, $p=.0401$ ) with females showing higher FC than males in the right hemisphere; however, post hoc $t$ tests did not confirm significant differences between means. No other significant main or interaction effects were detected.
When sex was excluded from the LMM model, the main effect of age remained significant $\left(F_{(3,45.2)}=3.1, p=.0363\right)$.

Amygdala-ACC (BA 24) FC. A significant age by group by sex interaction effect $\left(F_{(2,42.3)}=3.7, p=.0337\right)$ was detected in amygdala-ACC FC (Figure 1b). Post hoc $t$ tests, showed significantly different functional coupling between female groups at 12 months of age $\left(t_{(1,3.9)}=3.2, p=.0334, \eta^{2}=0.535\right)$, with positive $\mathrm{FC}$ in control females but negative FC in maltreated females. No other significant main or interaction effects were detected.

When sex was excluded from the LMM model no main or interaction effects were detected.

\section{Amygdala-OFC FC}

Amygdala-BA 11 FC. Apart from a trend effect of hemisphere $\left(F_{(1,42.6)}=3.2, p=.0820\right)$ with higher positive $\mathrm{FC}$ seen in the right than left hemisphere (Figure 1a), no significant main or interaction effects were detected.

When sex was excluded from the LMM model, a main effect of hemisphere was detected $\left(F_{(1,90.8)}=5.4, p=.0225\right)$.

Amygdala-BA 13 FC. A main effect of group $\left(F_{(1,60.0)}=5.0\right.$, $p=.0289$ ) was detected, with higher positive FC in control than maltreated animals (Figure 1a), and a trend for Group $\times$ Age $\left(F_{(3,41.3)}=2.7, p=.0573\right)$. Post hoc $t$ tests revealed significant differences at 3 months, with controls showing stronger (positive) FC than maltreated animals, who showed either uncoupling or negative FC $\left(t_{(1,32)}=3.83, p=.001, \eta^{2}=0.298\right)$. No other significant main or interaction effects were detected.

When sex was excluded from the LMM model, the main effect of group remained $\left(F_{(1,77.9)}=6.7, p=.0117\right)$, a significant main effect of age emerged $\left(F_{(3,50.5)}=3.5, p=.0216\right)$ and the group by age interaction effect remained $\left(F_{(3,47.5)}=2.8, p=.0474\right)$.

\section{Amygdala-dIPFC FC}

Amygdala-BA 9 FC. A significant age by group by hemisphere interaction effect $\left(F_{(3,36.7)}=4.3, p=.0106\right)$ was detected. Post hoc tests found significant differences between groups at 12 months, with positive FC in the left and negative in the right hemisphere of controls, whereas maltreated animals had negative FC in the left and positive in the right hemisphere $\left(t_{(1,6)}=4.2\right.$, $p=.0056, \eta^{2}=0.50$; Figure 1a). A significant age by sex by hemisphere interaction effect $\left(F_{(3,36.4)}=3.8, p=.0189\right)$ and trend for group by sex $\left(F_{(1,44.7)}=3.6, p=.0654\right)$ were also detected. No other significant main or interaction effects were found.

When sex was excluded from the LMM model, a main group effect emerged $\left(F_{(1,42.2)}=4.8, p=.0340\right)$, with controls showing weaker positive FC than maltreated animals.

Amygdala-BA 46 FC. A main effect of $\operatorname{sex}\left(F_{(1,74.5)}=4.8\right.$, $p=.0318)$ was found, with higher positive FC in females than males (data not shown in Figure 1). No other significant main or interaction effects were detected.

When sex was excluded from the LMM model there was a trend for an age by group interaction effect $\left(F_{(3,47.7)}=4.8\right.$, $p=.0842)$.

\section{Whole-brain voxel-wise amygdala FC}

Amygdala seed-based FC maps were FDR-corrected $(q=0.05$, $t>2.630, p<.0085)$, and clusters with ten or more contiguous voxels (faces touching) were selected to increase stringency for 
(a)
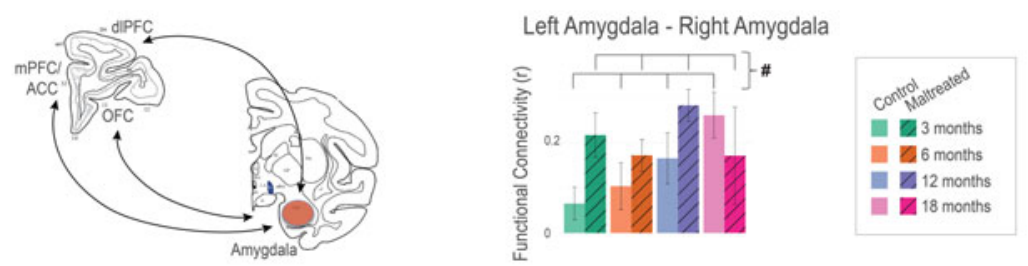

$\underline{\text { Left }}$

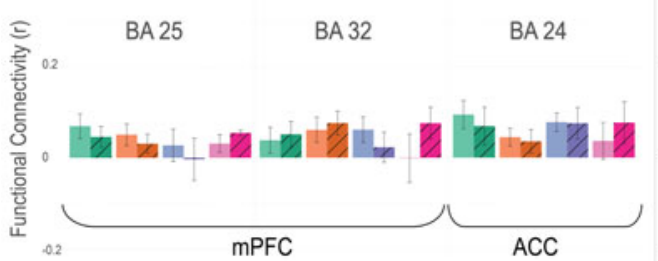

$\underline{\text { Right }}$
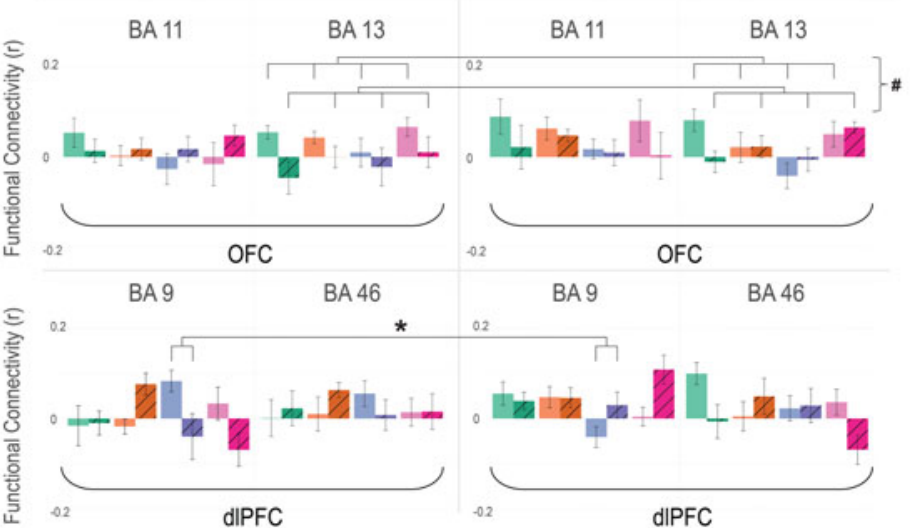

(b)
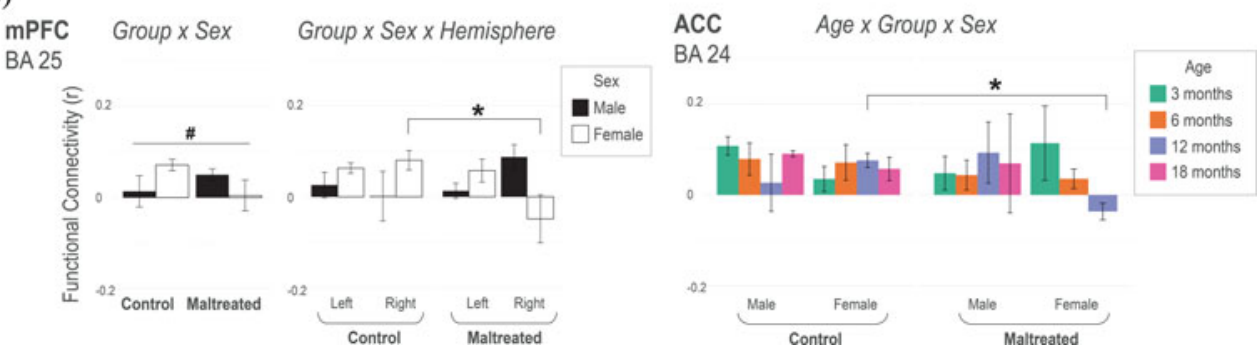

Figure 1. Development of amygdala-prefrontal cortex (PFC) functional connectivity (FC) from infancy through the juvenile period (3-18 months of age): region of interest (ROI)-ROI analysis. (a) Amygdala-amygdala and amygdala-PFC subregions FC across four ages are plotted separately by group, PFC subregion, respective Brodmann Areas (BA) and hemisphere: macaque amygdala and PFC coronal diagrams showing ROIs (adapted from Barbas, Saha, Rempel-Clower, \& Ghashghaei, 2003) - top, left; amygdala-amygdala FC was higher in maltreated than control animals across ages $\left(\#:\left(F_{(1,20.4)}=4.4, p=.0496\right)\right.$-top, right, whereas amygdala $F C$ with BA13 was stronger in control than maltreated animals $\left(\#: F_{(1,60.0)}=5.0, p=.0289\right)$-third row from top-; an age by group by hemisphere effect was detected for amygdala-BA9 FC $\left(F_{(3,36.7)}=4.3, p=.0106\right)$, with positive $\mathrm{FC}$ in the left and negative in the right hemisphere of controls, and opposite coupling in maltreated animals at 12 months $\left({ }^{*}: \mathrm{t}_{(1,6)}=4.2, p=.0056\right)$-fourth row from top. (b) Significant interaction effects of: group by sex on amygdala-medial PFC (mPFC) FC (BA 25; \#: $F_{(1,48.3)}$ $=5.3, p=.0258$ ), with higher FC in control than maltreated females, though post hoc tests did not confirm significant differences - first from left; group by sex by hemisphere on amygdala-BA $25 \mathrm{FC}\left(F_{(1,48.3)}=6.1, p=.0169\right)$ with higher positive $\mathrm{FC}$ in control than maltreated females in the right hemisphere $\left({ }^{*}: \mathrm{t}_{(1,12.0)}=2.3, p\right.$ $=.0431)$-second from left-; and age by group by sex for amygdala-ACC (BA 24) FC $\left(F_{(2.42 .3)}=3.7, p=.0337\right)$, showing different coupling between female groups at 12 months $\left({ }^{*}: \mathrm{t}_{(1,3.9)}=3.2, p=.0334\right)$, with positive FC in control, and negative FC in maltreated females -right. ACC: anterior cingulate cortex; dIPFC: dorsolateral PFC; mPFC: medial PFC; OFC: orbitofrontal cortex. Plots represent mean \pm standard error of the mean (SEM).

the secondary analyses (Figure 2b). The amygdala FC maps obtained in this infant and juvenile macaque dataset during development closely resemble those published in adult macaques (Amaral \& Price, 1984; Grayson et al., 2016; Reding et al., 2020; Russchen, Bakst, Amaral, \& Price, 1985). Thus, significant positive amygdala FC - red to yellow voxels in Figure $2 \mathrm{~b}$ - was found throughout regions in the temporal lobe, including bilateral amygdala and hippocampal regions, and PFC subregions and
ACC (consistent with the mostly positive AMY-PFC/ACC FC detected in the ROI-ROI analysis; Figure 1), subcortical regions (e.g., thalamus, caudate, and ventral striatum, including nucleus accumbens, and hypothalamus), cerebellum, and brainstem areas. Negative amygdala FC was found dispersed in the posterior cingulate cortex and cerebellum, as well as throughout motor, posterior parietal, and occipital (visual) cortices (blue voxels in Figure 2b). 


\section{(a) Amygdala Region of Interest}

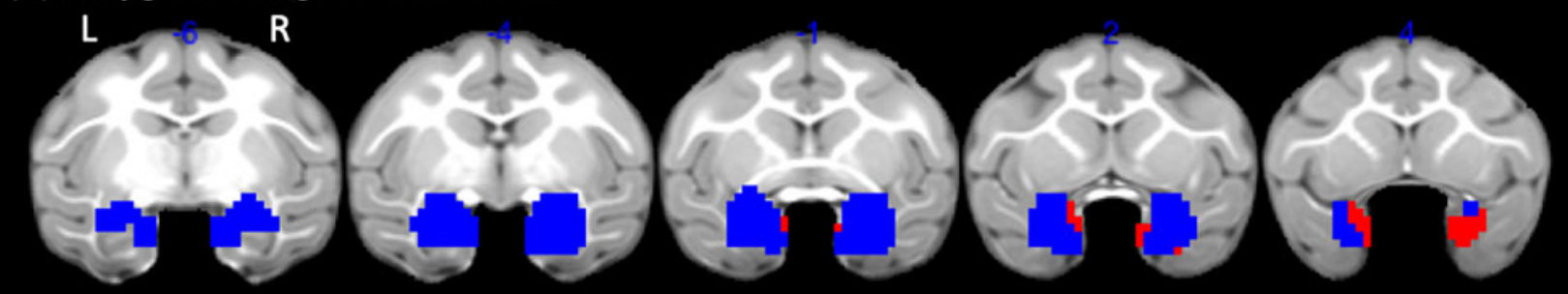

\section{(b) Amygdala Whole-Brain FC}
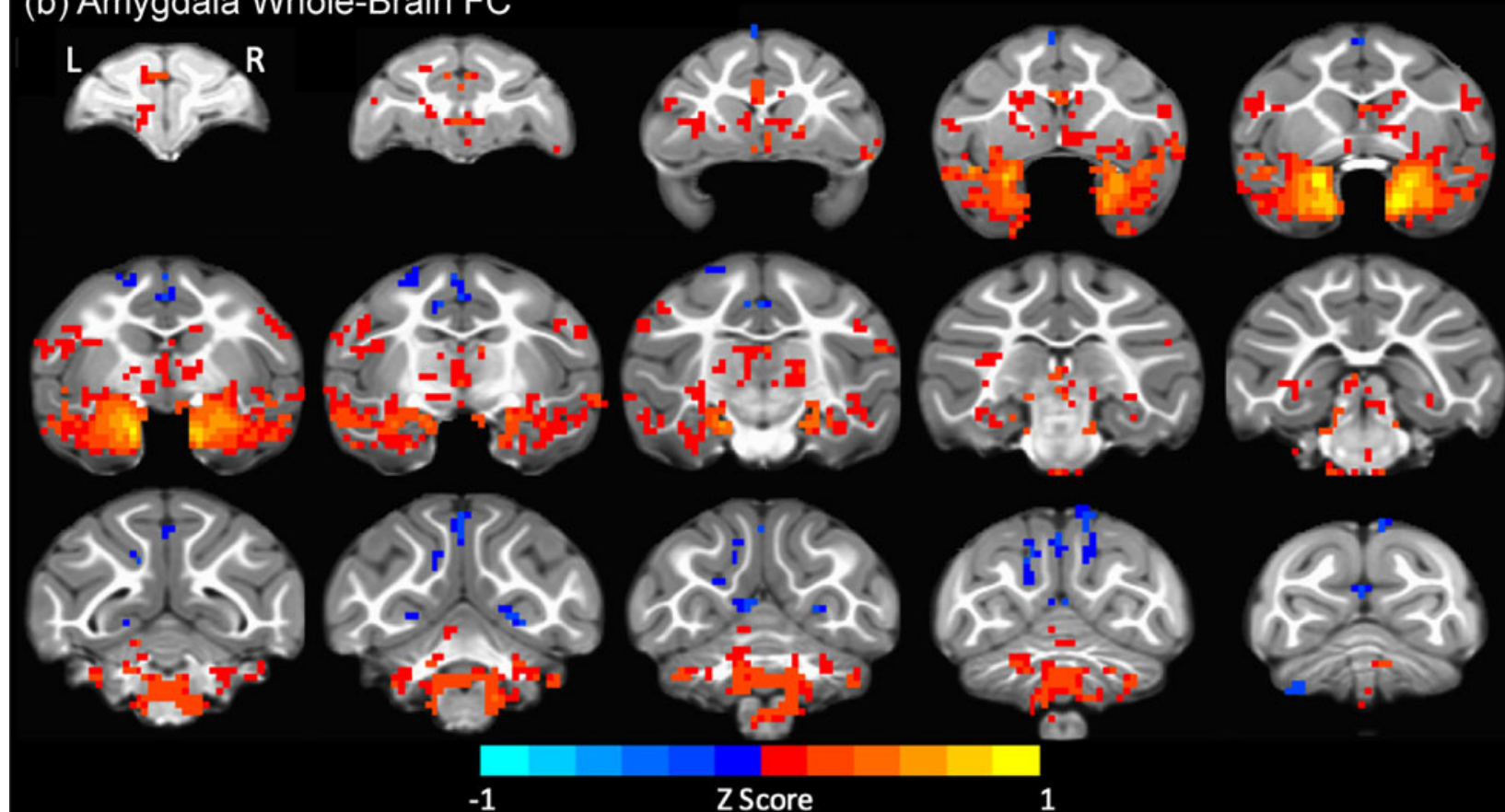

Figure 2. Amygdala functional connectivity $(\mathrm{FC})$ with the rest of the brain. (a) Amygdala region of interest (ROI) in blue, after removing voxels with blood oxygen level dependent (BOLD) signal dropout (in red). (b) Amygdala FC maps obtained from the seed-based whole-brain analysis collapsed across group, age and hemisphere (red to yellow voxels: positive FC; blue voxels: negative FC). L: left; R: right.

Secondary MLM (AFNI 3dLME) analysis restricted to these significant clusters of contiguous voxels selected from the amygdala FC maps showed a main effect of age that primarily reflected increased positive FC with age between amygdalae and bilateral corticomedial/medial amygdaloid nucleus, right anterior amygdaloid area; cortical regions, such as bilateral superior temporal sulcus (STS: TE anteroventral, TE anterodorsal, temporo-parieto- occipital junction), left parainsular cortex, left visual cortex (V1), left posterior cingulate, left perirhinal cortex, left entorhinal cortex, right periamygdaloid cortex and bilateral ventral/dorsal tegmental area, bilateral dorsal raphe nuclei, left lateral parabrachial nucleus, bilateral cerebellum, bilateral laterodorsal/lateroventral amygdala, left trigeminus, left abducens/facial nucleus/pontine reticular formation, and left pre/pro/parasubiculum (hippocampus) (FDR-corrected $q=0.05, t>5.242, p<.0023$, cluster threshold $\geq 2$ voxels; Figures $3 \& 4$ ).

Although there were no main effects of maltreatment, a caregiving group by age interaction effect was detected in amygdala FC with two main clusters: the left locus coeruleus (LC)/laterodorsal tegmental area (LDTA) and left periamygdaloid cortex (PAC)/basal amygdala (BA). Maltreatment effects emerged with age so that, while controls' FC strengthened between 12 and 18 months, maltreated animals' FC with LC/LDTA weakened (became uncoupled) or became negatively coupled with PAC/ BA (FDR-corrected $(q=0.05), t>7.146, p<2.5 \times 10^{-4}$, cluster threshold $\geq 2$ voxels; Figure 5). Post hoc ( $t$ test) comparison of the means confirmed controls had stronger FC with both clusters than maltreated animals at 18 months $\left(\mathrm{PAC} / \mathrm{BA} t_{(5.9)}=4.47\right.$, $p=.0043, \eta^{2}=0.44 ;$ LC/LDTA $\left.t_{4.3}=3.7, p=.0179, \eta^{2}=0.24\right)$ and also at 12 months for the LC/LDTA $\left(t_{(11)}=2.49, p=.0302 ; \eta^{2}=\right.$ $0.07)$ but not PAC/BA $\left(t_{(10.3)}=-0.36, p=.7274, \eta^{2}=0.002\right)$. Although not significant based on post hoc comparisons, LC/ LDTA seems higher in maltreated than controls initially (at 3 months), followed by gradual decoupling at later ages.

\section{Associations between postnatal cortisol exposure and maltreatment effects on amygdala FC}

Associations with amygdala-PFC FC from ROI-ROI analysis Linear regression models were run to test whether hair cortisol concentrations at 6 months predicted amygdala-PFC FC group effects detected in the LMM analysis. Hair cortisol at 6 months significantly predicted amygdala-dlPFC (BA9) at 12 months in 


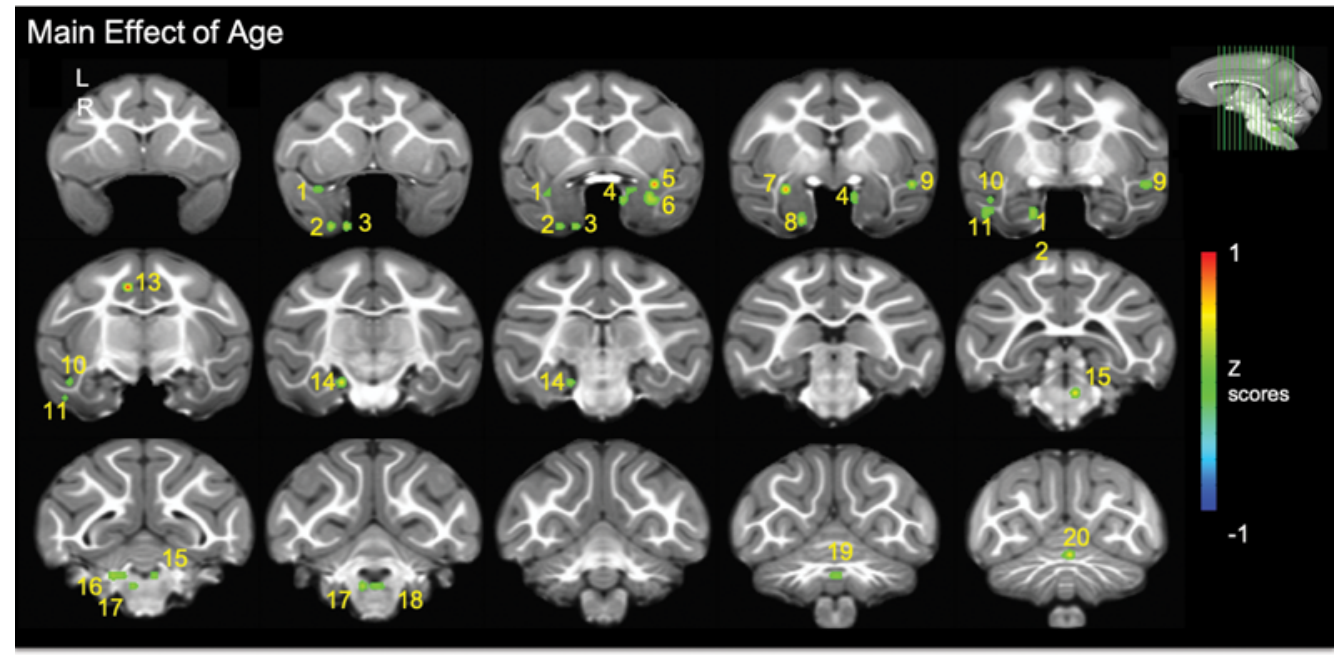

Figure 3. Voxel-wise analysis of developmental changes in amygdala FC with the rest of the brain (3-18 months of age). Amygdala functional connectivity (FC) obtained from the seed-based, whole-brain analysis. Clusters with significant main effect of age, false discovery rate (FDR) corrected ( $q=0.05)$ : $1-$ Parainsular cortex, $2 \& 3$ - Perirhinal cortex,4 - Corticomedial/medial amygdaloid nucleus, 5 - Anterior amygdaloid area, 6 - Laterodorsal/lateroventral amygdala, 7 Laterodorsal amygdala, 8 - Perirhinal cortex, 9 - TPO/STS, 10 - TEa, STS ventral bank, 11 - Teav, Tead, 12 - Presubiculum/Prosubiculum, 13 - Posterior Cingulate, 14 - Parasubiculum, 15 - Dorsal \& Laterodorsal tegmental area, 16 - Lateral parabrachial complex, 17 - Principal sensory, 18 - Dorsoventral tegmental area/dorsal Raphe-caudal nuclei, 19\&20 - Cerebellum. L: left; R: right.

the right hemisphere $\left(\beta=0.0036, F_{(1,5)}=6.6, p=.0497\right.$; adjusted $R^{2}=0.48$; Figure $\left.6 \mathrm{a}\right)$; higher cortisol was associated with a switch from negative to positive coupling. No other significant associations were found between postnatal cortisol exposure and amygdala-PFC FC.

\section{Associations with whole brain voxel-wise amygdala FC}

Linear regression models were run to test whether hair cortisol concentrations at 6 months predicted the maltreatment effects identified in the MLM (AFNI 3dLME) analysis on amygdala FC with PAC/BA and LC/LDTA clusters at 18 months of age. Hair cortisol at 6 months significantly predicted amygdala FC with the PAC/BA cluster $\left(\beta=-0.0027, F_{(1,5)}=8.2, p=.0351\right.$; adjusted $R^{2}=$ $0.56)$ and with the LC/LDTA cluster $\left(\beta=-0.0028, F_{(1,5)}=-8.7\right.$, $p=.0003$; adjusted $R^{2}=0.9$ ) at 18 months (Figure $6 \mathrm{~b}$ ).

\section{Discussion}

The goal of the current study was to determine the developmental impact of infant maltreatment on amygdala FC longitudinally, from macaque infancy through the juvenile (prepubertal) period, which may underlie the enhanced emotional and stress reactivity reported previously by our group in maltreated animals. This study utilized a well-established rhesus monkey model of infant maltreatment by the mother leading to infant distress. RsfMRI scans were collected longitudinally on these animals during infancy (at 3 and 6 months of age) and during the juvenile prepubertal period (at 12 and 18 months of age). ROI-ROI analyses examined specific alterations in amygdala-PFC circuit FC that could explain the higher emotional reactivity, anxiety, and stress neuroendocrine activity reported in these maltreated animals (Bramlett et al., 2017, 2018; Drury et al., 2017). From infancy to the juvenile period, maltreated animals showed weaker amygdala FC with subgenual cingulate (BA 25) and ACC (BA 24) than controls -although only in females - and with OFC (BA 13) and dlPFC (BA 9) in both sexes. Stronger FC was also found between left and right amygdala in maltreated than control animals. The amygdala FC voxel-wise analysis detected developmental increases with age with many regions, likely reflecting increased processing of socioemotionally relevant stimuli from infancy to the juvenile period. It also identified maltreatment effects on amygdala FC that emerged with age. Thus, whereas controls' amygdala functional coupling with regions implicated in arousal and stress (i.e., LC/LDTA) increased during the juvenile period, it became weaker in maltreated animals, and was predicted by higher cortisol exposure during infancy. These findings suggest that maternal maltreatment results in developmental alterations of amygdala circuits that include a weakened amygdala FC with PFC and brainstem arousal centers, some of them predicted by exposure to elevated cortisol levels during infancy. Future studies are needed to identify the cellular and molecular mechanisms underlying the dynamic developmental changes and alterations we have uncovered in theses circuits, which could generate useful neural biomarkers for future studies testing interventions in individuals with childhood maltreatment and other adverse experiences.

Our exploratory, seed-based voxel-wise analysis showed, first of all, that amygdala FC with PFC subregions and the ACC (BA 24) are positively coupled, which is consistent with the findings from the ROI-ROI analysis. In contrast to a previous study in adult macaques reporting absence of negative FC between amygdala and other regions of the brain (Grayson et al., 2016), we found negative FC with the posterior cingulate cortex, cerebellum, and motor, posterior parietal, and occipital (visual) cortices. These findings are consistent with other reports in adult macaques (Reding et al., 2019), including a lack of negative FC between amygdalae and hypothalamus. Our results are also consistent with studies in adult humans reporting positive amygdala FC with regions throughout the temporal lobe, bilateral amygdalae and hippocampal regions, subcortical regions (e.g., thalamus and caudate), insula, and brainstem, and negative FC with cerebellum, and occipital and parietal lobes (Roy et al., 2009).

Secondly, the seed-based analyses showed brain wide strengthening of amygdala FC with many regions in the brain as infants mature. This may support increased processing of social and 


\section{Connectivity between Amygdalae and Left Parainsular Cortex}

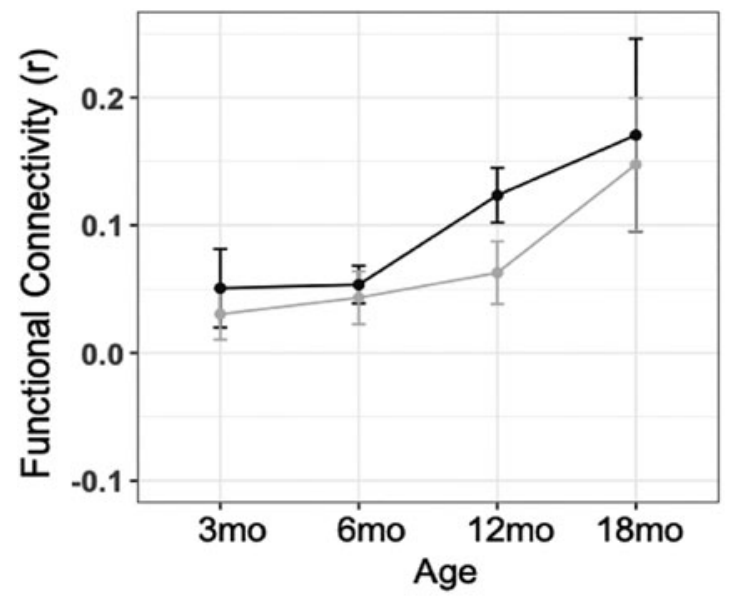

\section{Connectivity between Amygdalae and Right Ventral/Dorsal Tegmental Area/Dorsal Raphe - caudal nuclei}

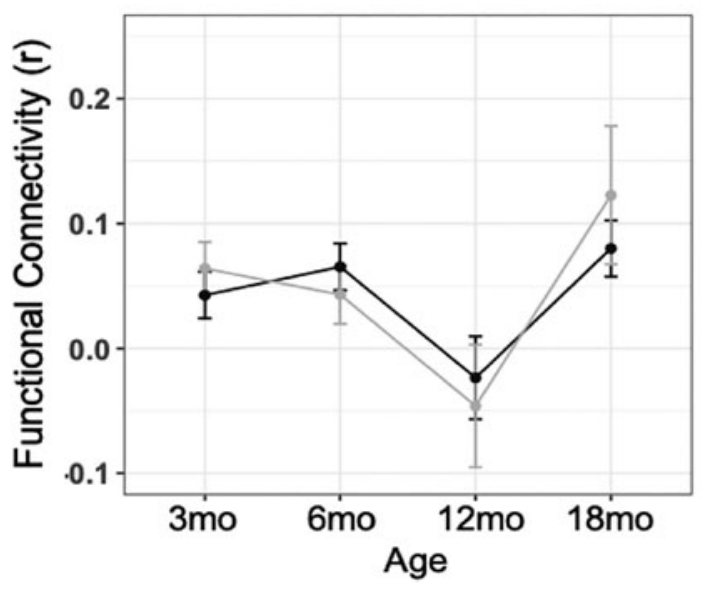

\section{Connectivity between Amygdalae and Right TPO/Superior Temporal Sulcus}

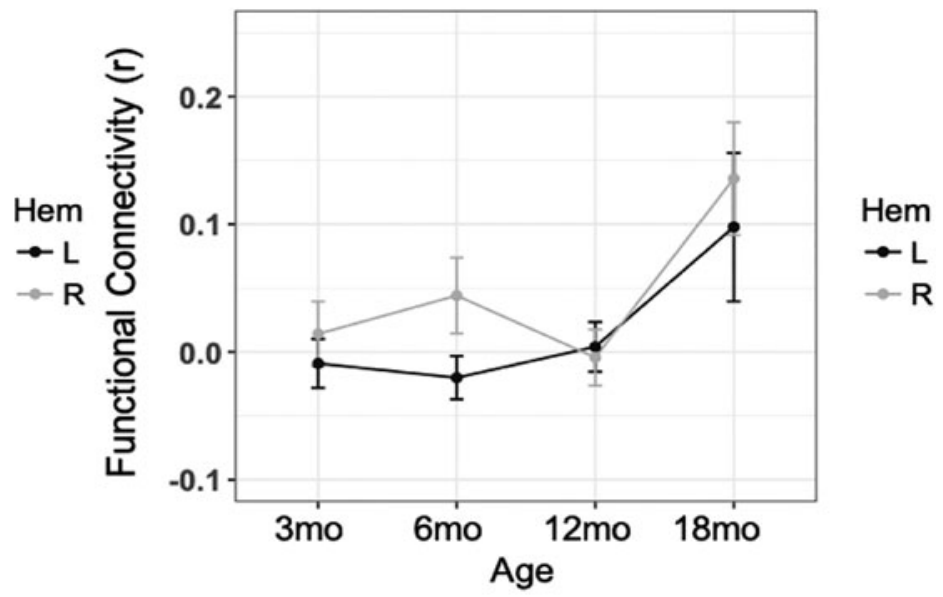

\section{Connectivity between Amygdalae and Left Perirhinal Cortex}
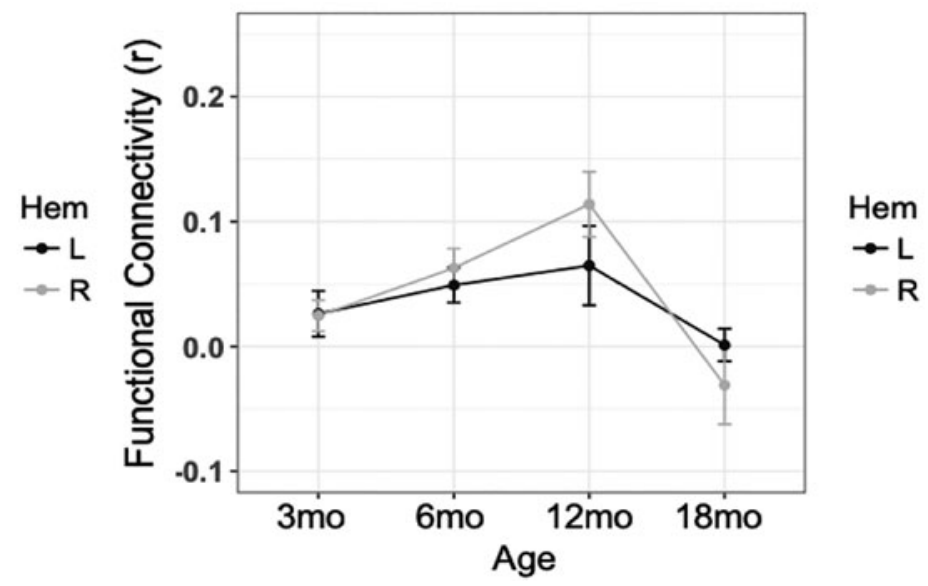

Figure 4. Representative examples of brain clusters with significant developmental changes in functional connectivity (FC) with amygdala. FC between right/left amygdala and four representative clusters showing a significant main effect of age. L: left; R: right.

emotional stimuli as the infants mature, especially in relation to the emergence of threat and fear responses as the animals go through weaning (3-6 months), and increased exploration, play with peers, and independence from the mother, particularly during the juvenile period (12, 18 months) (Sanchez et al., 2015). Similar changes in amygdala FC have already been mapped to amygdala functional maturation during weaning in rat pups (Sullivan, Landers, Yeaman, \& Wilson, 2000). The developmental changes in amygdala FC were also region-specific, including certain amygdaloid nuclei, regions along the ventral visual pathway and other temporal sensory areas, and the cerebellum.

Some of the discrepancies between our voxel-wise analysis results and other macaque reports in relation to positive versus negative FC valence might be explained by developmental changes in amygdala FC with these regions. Among regions showing a main effect of age, amygdala FC increased with age in regions of the ventral visual pathway (STS, TEav, TEad, $\mathrm{TPO}$ ), which may support the emergence of face processing and facial expression recognition during the first few months of life (Kuwahata, Adachi, Fujita, Tomonaga, \& Matsuzawa, 2004; Lutz, Lockard, Gunderson, \& Grant, 1998; Muschinski et al., 2016; Parr et al., 2016; Sugita, 2008). Interestingly, prior to 18 months of age, amygdala FC with right TPO/STS was close to zero, consistent with previous reports in children (Gabard-Durnam et al., 2014), and interpreted by the authors as functional uncoupling between these regions, which becomes stronger during the juvenile period when higher-order face processing has matured and inputs from the amygdala are pruned and refined (Webster, Ungerleider, \& Bachevalier, 1991a, 1991b) to inform on the valence of facial expressions.

Amygdala FC with the cerebellum was found to decrease with age, in contrast to what has been reported in humans, where a strengthening between centromedial amygdala (CMA) and the cerebellum emerges with age (Qin, Young, Supekar, Uddin, \& Menon, 2012), as the CMA and its connections regulate reflexive and defensive behaviors in response to fear (LeDoux, 2000, 2007). Other 


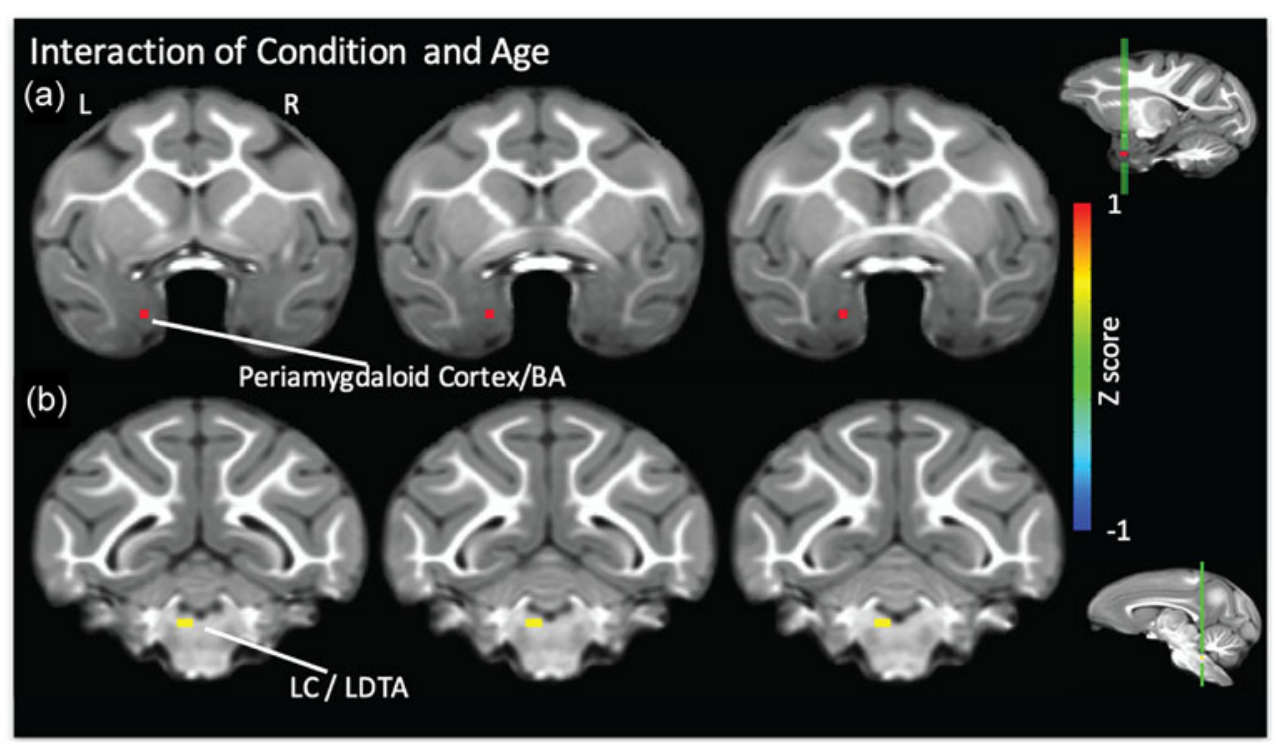

(c)
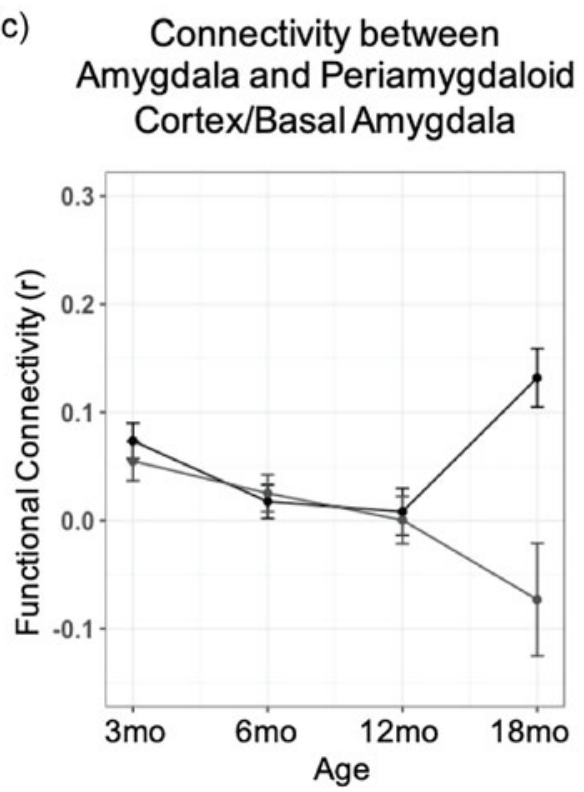

Connectivity between

Amygdala and Locus

Coeruleus/Laterodorsal

Tegmental Area

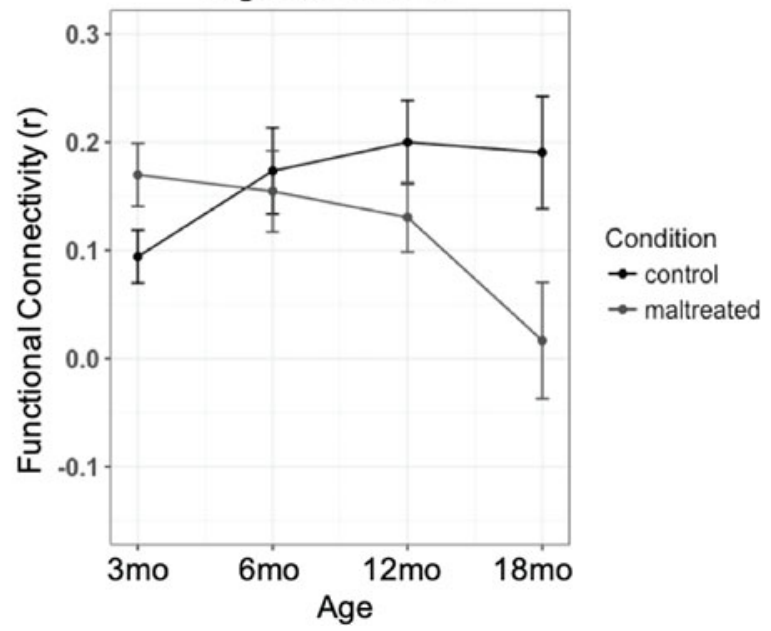

Figure 5. Brain clusters showing a significant group (condition) by age interaction effect on functional connectivity with amygdala. (a) Periamygdaloid cortex/basal amygdala (PAC/BA). (b) Locus coeruleus (LC)/laterodorsal tegmental area (LDTA). L: left; R: right. (c) Developmental trajectories of amygdala FC with these two clusters show maltreatment effects emerging with age (between 12 and 18 months) so that, while control animals' FC strengthens, maltreated animals' FC with LC/LDTA weakens (becomes uncoupled) or becomes negatively coupled with PAC/BA (left).

regions involved in sensory processing and memory (entorhinal cortex, parasubiculum, perirhinal cortex) also showed decreased FC with the amygdala with age in our animals, although it has been reported to be strong in adulthood, especially amygdalaBLA (basolateral amygdala). Perhaps this is another circuit that is still developing and may "switch" and become stronger during adolescence and adulthood (Qin et al., 2012), as this connectivity is important for detecting and perceiving fearful stimuli.

Our seed-based analysis also showed a maltreatment by age interaction effect in the left LC/LDTA and left PAC/BA, regions implicated in arousal, vigilance, and fear learning, with maltreatment effects emerging with age. Thus, while controls' amygdala FC with LC/LDTA and PAC/BA strengthened between 12 and 18 months, maltreated animals' FC weakened (became uncoupled) between
amygdala-LC/LDTA or became negatively coupled with PAC/BA. Interestingly, FC with LC/LDTA seemed initially higher in maltreated than controls (at 3 months), which parallels the hyperarousal and reactivity observed during infancy, followed by gradual decoupling of the two regions at later ages (12 and 18 months: juvenile period). Although this is speculative, such decoupling could potentially serve an adaptive role to downregulate the arousal system, as reported in some studies with maltreated children (McLaughlin et al., 2016).

Our finding that hair cortisol at 6 months is predictive of FC in LC/LDTA and PAC/BA clusters at 18 months of age, although from a very small subset of animals, is consistent with previous reports of effects of cortisol/glucocorticoid exposure on FC. Exposure to chronic stress and/or glucocorticoids affects spine density and dendritic arborization, in circuitry vulnerable to the 
Right Amygdala - dIPFC (BA 9) at 12mo \& Hair Cortisol at $6 \mathrm{mo}$

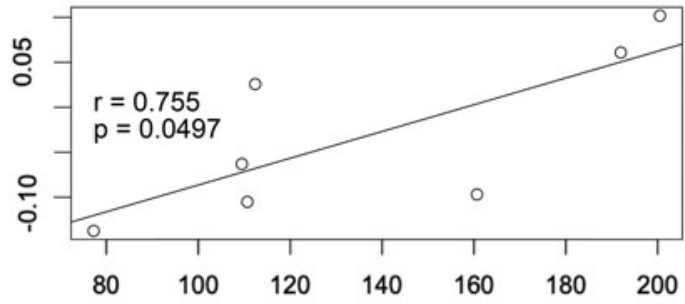

Hair Cortisol (pg/mg)

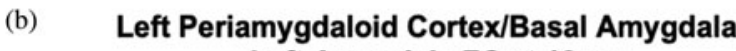
- Left Amygdala FC at 18mo \& Hair Cortisol at $6 \mathrm{mo}$

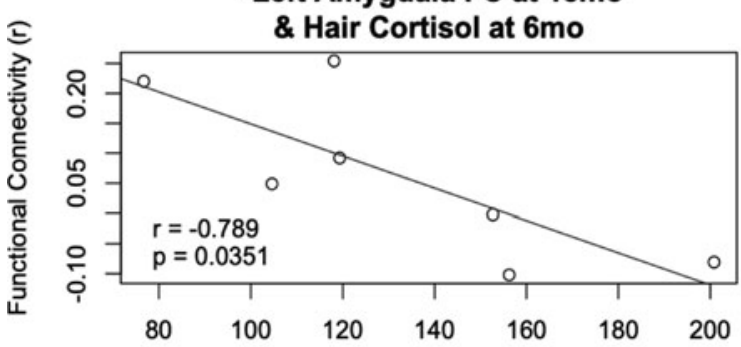

Hair Cortisol (pg/mg)

Left Lateral Dorsal Tegmental Area/Locus Coeruleus - Left Amygdala FC at 18mo

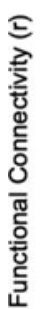
\& Hair Cortisol at $6 \mathrm{mo}$

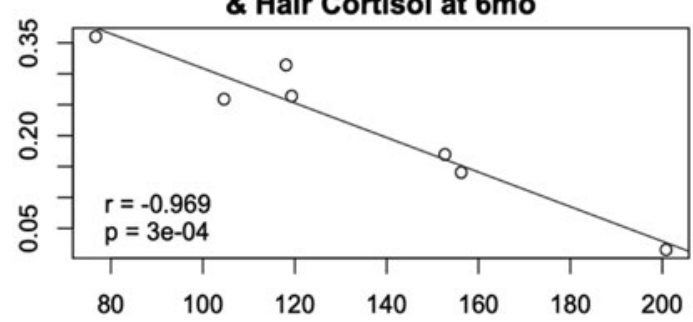

Hair Cortisol (pg/mg)

Figure 6. Associations between hair cortisol levels at 6 months and amygdala functional connectivity (FC). Higher hair cortisol accumulation from birth through 6 months predicts: (a) switch from negative to positive coupling between dorsolateral prefrontal cortex (dIPFC) (BA 9) and the amygdala; (b) weaker FC between periamygdaloid cortex/basal amygdala and locus coeruleus (LC)/laterodorsal tegmental area (LDTA) clusters and the amygdala.

effects of trauma (McEwen, 2004; Roozendaal, McEwen, \& Chattarji, 2009; Vyas, Mitra, Shankaranarayana Rao, \& Chattarji, 2002). Some of these regions, such as PFC, the amygdala, and hippocampus, have high levels of glucocorticoid receptors, which suggests they are particularly sensitive to chronic exposure to cortisol (Anisman, Zaharia, Meaney, \& Merali, 1998; Sanchez, Milanes, Pazos, Diaz, \& Laorden, 2000a; Sanchez, Young, Plotsky, \& Insel, 2000b). FC studies in adult men given a one-time dose of hydrocortisone, have also reported uncoupling of the amygdala, especially a reduction in positive FC with regions involved in initiating and maintaining the stress response, such as the $\mathrm{LC}$, in addition to reducing negative FC with regions involved in executive functions (Henckens, van Wingen, Joels, \& Fernandez, 2012). Thus, exposure to chronically high glucocorticoid levels during early development (as we previously reported in (Drury et al., 2017) could initiate effects that may last into the juvenile period in maltreated animals, through such FC disconnection between amygdala-LC/LDTA and -PAC/ BA clusters.

Cortisol fluctuations occur normally across the circadian rhythm and during ultradian oscillations on a smaller temporal scale, aiding in the stabilization of spines and pruning of preexisting synapses, a balance important in learning and plasticity (Liston et al., 2013). However, exposure to high levels of glucocorticoids, even as little as $30 \mathrm{~min}$ of increased exposure (Chen, Dube, Rice, \& Baram, 2008), prevents the formation of new spines and pruning of synapses during development, a time of enhanced plasticity and remodeling (Liston \& Gan, 2011). Deficits in spine stabilization, formation, maintenance, and synaptic pruning during this period of development could lead to reduced structural connectivity of amygdala circuits, potentially resulting in a weaker FC of the amygdala to LC/LDTA and PAC/BA, and may mediate increased synaptic transmission in thalamic sensory neurons (by way of activating cholinergic projections), during states of fear. In studies of early developmental exposure to glucocorticoids in rodents, increased recruitment of cholinergic neurons in the LDTA can lead to prolonged hyperanxious states (Borges et al., 2013; Kaufer, Friedman, Seidman, \& Soreq, 1998). This "programming" of LDTA neurons towards increased activity could lead to heightened arousal and vigilance reported in maltreated animals despite weakened FC with the amygdala. Rodent studies have shown that cholinergic neurons in the LDTA also project to the LC (Jones \& Yang, 1985). Activation of these terminals through perfusions of cholinoceptor agonists in the LC increase neuronal firing rate and arousal, suggesting that the LDTA projections to the LC are bolstering arousal through excitatory connections (Egan \& North, 1985; Engberg \& Svensson, 1980). In response to stressful stimuli, LC neurons are activated (Abercrombie \& Jacobs, 1987; Aston-Jones, Chiang, \& Alexinsky, 1991; Grant, Aston-Jones, \& Redmond, 1988; Rasmussen, Morilak, \& Jacobs, 1986) and facilitate norepinephrine increase in the amygdala through terminals in the BLA (Asan, 1998). This pathway is thought to support proper neuroendocrine responses to a stressor or fearful stimulus. Reciprocal connections from the amygdala to the LC (Van Bockstaele, Bajic, Proudfit, \& Valentino, 2001) provide feedback to facilitate an increase in responding to stressful stimuli, and therefore arousal (Goldstein, Rasmusson, Bunney, \& Roth, 1996), which may be implicated in the development of maladaptive responses to stress in fear disorders (Buffalari \& Grace, 2007). Norepinephrine (NE) projections to the amygdala also modulate memories of aversive stimuli (McGaugh, 2002) through consolidation of learning within the BLA (Gallagher, Kapp, Musty, \& Driscoll, 1977). Thus, a weakened amygdala FC (or uncoupling) of this pathway in maltreated animals, could potentially contribute to abnormal regulation of fear learning to specific cues, and noise in this circuit during memory consolidation, leading to increased generalized fear and stress responses.

Corticotropin releasing factor (CRF), a peptide hormone involved in the stress response, also plays a role in the LC NE system. In response to chronic stress, there is a postsynaptic sensitization to CRF in the LC, leading to an increased release of NE to downstream targets (Finlay et al., 1997). This plasticity may result in hyperactivity or hyperresponsiveness of the LC-NE system. As detailed above, disconnection between amygdala and LC could result from exposure to chronic stress-induced increased cortisol, as previously shown in human studies (Henckens et al., 2012). One could speculate that such amygdala decoupling could be adaptive, downregulating the arousal system and resulting in 
blunted emotional or physiological responses prioritizing activity in other networks to avoid overwhelming the system (or even helping normalize the HPA axis activity). Indeed, blunted physiological responses (skin conductance [SCR] and heart rate acceleration) to threatening stimuli have been reported in children and adolescents with a history of childhood maltreatment (Busso, McLaughlin, \& Sheridan, 2017; Machlin, Miller, Snyder, McLaughlin, \& Sheridan, 2019; MacMillan et al., 2009; McLaughlin et al., 2016; Ouellet-Morin et al., 2011; Trickett, Gordis, Peckins, \& Susman, 2014), as well as in adults that experienced trauma across the lifespan, in parallel to blunted startle responses (D'Andrea, Pole, DePierro, Freed, \& Wallace, 2013; Heleniak, McLaughlin, Ormel, \& Riese, 2016; Lovallo, Farag, Sorocco, Cohoon, \& Vincent, 2012; McTeague \& Lang, 2012), supporting our speculative interpretation. Interestingly, these blunted physiological responses have been reported in fear conditioning paradigms, where maltreated children exhibit reduced SCR towards the fear stimulus (CS+), and do not show a dissociable SCR between fear/safety stimuli (CS+/CS-) (McLaughlin et al., 2016). This may suggest deficits in fear learning/associative learning and is consistent with blunted fear conditioning responses and impaired discrimination of fear/safety stimuli we have identified in our maltreated animals when they reach adolescence (Morin et al., 2018).

Regarding the results of the amygdala-PFC ROI-ROI analysis, weaker FC was found between amygdala and ACC/mPFC in maltreated animals, particularly in females (i.e., weaker amygdala FC with $\mathrm{mPFC}$ regions: BA 25 (subgenual cingulate), BA 24 (ACC)) as well as with dlPFC region BA 9, and OFC region BA 13. These findings are consistent with previous reports in human populations with early adverse experiences (VanTieghem \& Tottenham, 2018), including adolescents and adults with histories of childhood maltreatment (Thomason et al., 2015) and in humans with PTSD (Fonzo et al., 2010b). A weak amygdalamPFC FC is linked to impaired stress and emotional regulation, and can contribute to arousal dysregulation through hypervigilance and attention bias to threat.

Weaker FC between amygdala and left periamygdala/basal amygdala in maltreated than control animals also emerged at the later age (18 months). In fact, at this age controls' FC gets stronger (positive) and maltreated animals' FC becomes uncoupled. The periamygdaloid cortex receives direct connections from the olfactory bulb and is involved in conditioning to explicit environmental sensory stimuli. The basal amygdala also plays a critical role in fear learning, specifically conditioned fear expression and extinction (Amano, Duvarci, Popa, \& Pare, 2011). Previous studies in humans have reported increased activation of the amygdala when subjects have prior awareness of the aversive nature of the stimuli (Morris, Ohman, \& Dolan, 1998). Perhaps the reduced FC between amygdalae subnuclei (such as the left basal amygdala) could interfere with the modulation of the fear response to learned aversion of environmental stimuli in maltreated animals.

We would also like to discuss briefly the potential interpretations of negative or anticorrelated FC. In previous studies, negative resting state $\mathrm{FC}$ has been shown to reflect competition between brain networks (i.e., default mode and task positive networks; (Kelly, Uddin, Biswal, Castellanos, \& Milham, 2008) or a history of regulation on one region over another (i.e., limbic circuits; (Liang, King, \& Zhang, 2012; Roy et al., 2009). However, some of the negative FC (correlations) between regions in our study are very close to zero, which may in fact be due to the regression of the global signal, which would center all correlations about zero, and not represent a true negative correlation. Additionally, near-zero correlations could be interpreted as an uncoupling of the amygdala with other regions in maltreated animals. Uncoupling or disconnection between limbic and regulatory regions, such as the amygdala and insula with the ACC, has been shown in individuals with PTSD when evaluating fearful/threatening stimuli (Fonzo et al., 2010a; Rockstroh \& Elbert, 2010).

Despite the strengths of this study, there are also important limitations. Notably, the sample size used in these analyses which, although large for a macaque study, is still small and lacks statistical power to investigate complex interactions between variables. Although likely underpowered, our findings do suggest strong sex differences in maltreatment effects and emphasize the importance of including sex as a factor in neurodevelopmental studies of childhood maltreatment. It would also be important to do a follow up, larger, study where the effects of maltreatment on animals reared by their biological mothers could be statistically compared with those on cross-fostered infants, and between males and females. Due to illness, image artifact or other factors not related to the study, not all animals had imaging data at all 4 ages $(3,6,12,18$ months), although the 3dLME model allowed to include animals that had at least two time points in the analysis. Despite limitations inherent to rsfMRI (in particular, the lack of directionality information in FC measures when using rsfMRI) it has strengths and advantages to task fMRI for developmental and translational purposes, and has proven useful to characterize brain function and emotional regulation across many human populations (Britton et al., 2013; Costa Dias et al., 2015; Foster \& Wilson, 2006; Gee et al., 2013; Hare et al., 2008; Matthews \& Fair, 2015). Due to the dampening effect of isoflurane on BOLD signal, we standardized levels to the lowest possible in the literature ( $1 \%$ isoflurane). Previous studies show robust and dynamic fluctuations of the BOLD signal and patterns of network FC similar to awake monkeys, even using $\geq 1 \%$ isoflurane anesthesia (Grayson et al., 2016; Hutchison et al., 2013; Hutchison et al., 2012; Li et al., 2013; Margulies et al., 2009; Miranda-Dominguez et al., 2014b; Palanca et al., 2015; Sallet et al., 2011; Tang \& Ramani, 2016; Vincent et al., 2007) and dose-dependent effects of isoflurane on BOLD signal at much higher dosages (Hutchison, Hutchison, Manning, Menon, \& Everling, 2014). Finally, although our amygdala-based voxel-wise FC analyses followed data-reduction methods similar to previous studies in macaques (Grayson et al., 2016; Reding et al., 2020), limiting the analysis to voxels that survive a significant FC threshold collapsed across ages and groups for selection of amygdala FC maps might have resulted in false negatives.

In summary, our findings demonstrate that infant maltreatment alters the development of amygdala circuits, resulting in weakened amygdala FC with PFC and brainstem arousal centers, and in part predicted by exposure to elevated cortisol levels during infancy. It is possible that the derailed developmental trajectories in amygdala circuits have a downstream effect on other brain networks, which are being explored as these animals develop into adolescents and adults. Overall, these seem to be sensitive, promising and useful neural biomarkers for future studies and to test interventions in individuals with childhood maltreatment and other adverse experiences. Ongoing studies are being conducted to elucidate the behavioral correlates of the amygdala functional connectivity differences reported here; in particular, whether they do lead to changes in arousal, fear, and stress regulation. 
Acknowledgments. The authors want to thank Anne Glenn, Christine Marsteller, Dora Guzman and the staff at the Yerkes National Primate Research Center (YNPRC) and its Imaging Center for the excellent technical support and animal care provided during these studies. We also thank Drs. Melinda Higgins and Christa Payne for statistical guidance, Dr. Jocelyne Bachevalier for anatomical input on the regions of interest and the members of the "Early Experience, Stress and Neurobehavioral Development Center" (P50 MH078105) for the stimulating discussions of data presented here.

Funding Statement. This work was supported by funding from the National Institutes of Health (NIH) grants MH078105, MH078105-01S1, MH078105-04S1, MH091645, MH100029, DA038588, HD055255, MH901645, HD079124, F31 MH086203, T15LM007088, as well as by the NIH's Office of the Director, Office of Research Infrastructure Programs, P51OD011132 (YNPRC Base grant) and the Oregon Clinical and Translational Institute (CTSA UL1TR000128). The YNPRC is fully accredited by the Association for the Assessment and Accreditation of Laboratory Animal Care (AAALAC), International.

Conflicts of interest. None

\section{References}

Abercrombie, E. D., \& Jacobs, B. L. (1987). Single-unit response of noradrenergic neurons in the locus coeruleus of freely moving cats. I. Acutely presented stressful and nonstressful stimuli. Journal of Neuroscience, 7 , $2837-2843$.

Amano, T., Duvarci, S., Popa, D., \& Pare, D. (2011). The fear circuit revisited: Contributions of the basal amygdala nuceli to conditioned fear. Journal of Neuroscience, 31, 15481-15489.

Amaral, D. G., \& Price, J. L. (1984). Amygdalo-cortical projections in the monkey (Macaca fascicularis). Journal of Comparative Neurology, 230, 465-496. doi:10.1002/cne.902300402

Anisman, H., Zaharia, M. D., Meaney, M. J., \& Merali, Z. (1998). Do early-life events permanently alter behavioral and hormonal responses to stressors? International Journal of Developmental Neuroscience, 16, 149-164.

Asan, E. (1998). The catecholaminergic innervation of the rat amygdala. Advances in Anatomy, Embryology and Cell Biology, 142, 1-118.

Asok, A., Bernard, K., Roth, T., Rosen, J., \& Dozier, M. (2013). Parental responsiveness moderates the association between early-life stress and reduced telomere length. Development and Psychopathology, 25, 577-585. doi:10.1017/s0954579413000011

Aston-Jones, G., Chiang, C., \& Alexinsky, T. (1991). Discharge of noradrenergic locus coeruleus neurons in behaving rats and monkeys suggests a role in vigilance. Progress in Brain Research, 88, 501-520.

Barbas, H., Saha, S., Rempel-Clower, N., \& Ghashghaei, T. (2003). Serial pathways from primate prefrontal cortex to autonomic areas may influence emotional expression. BMC Neuroscience, 4, 25. Epub 2003/10/11. doi: 10.1186/ 1471-2202-4-25. PubMed PMID: 14536022. PMCID: PMCPMC270042.

Borges, S., Coimbra, B., Soares-Cunha, C., Ventura-Silva, A. P., Pinto, L., Carvalho, M. M., ... Sousa, N. (2013). Glucocorticoid programing of the mesopontine cholinergic system. Frontiers in Endocrinology (Lausanne), 4, 190. doi:10.3389/fendo.2013.00190

Bramlett, S., Morin, E. L., Guzman, D, Howell, B. R., Meyer, J. S., \& Sanchez, M. M. (2017). Effects of adverse maternal care on the development of hypothalamic-pituitary-adrenal (HPA) axis function in nonhuman primates. Paper presented at the 47th Annual Meeting of the Society for Neuroscience (SfN), Washington, DC.

Bramlett, S., Wakeford, A., Morin, E., Guzman, D. G., Siebert, E., Howell, B., ... Sanchez, M. M. (2018). Early hypothalamic-pituitary-adrenal axis activity predicts anxiety and sensitivity to the reinforcing effects of cocaine in adolescent macaques: early life stress as a risk factor. Paper presented at the 48th Annual Meeting of the Society for Neuroscience (SfN), San Diego, CA.

Brent, L., Koban, T., \& Ramirez, S. (2002). Abnormal, abusive, and stressrelated behaviors in baboon mothers. Biolofical Psychiatry, 52, 1047-1056.

Britton, J. C., Grillon, C., Lissek, S., Norcross, M. A., Szuhany, K. L., Chen, G., ... Pine, D. S. (2013). Response to learned threat: an fMRI study in adolescent and adult anxiety. American Journal of Psychiatry, 170, 1195-1204.
Buffalari, D. M., \& Grace, A. A. (2007). Noradrenergic modulation of basolateral amygdala neuronal activity: Opposing influences of alpha-2 and beta receptor activation. Journal of Neuroscience, 27, 12358-12366. doi:10.1523/jneurosci.2007-07.2007

Burgess, G. C., Kandala, S., Nolan, D., Laumann, T. O., Power, J. D., Adeyemo, B., ... Barch, D. M. (2016). Evaluation of denoising strategies to address motion-correlated artifacts in resting-state functional magnetic resonance imaging data from the human connectome project. Brain Connect, 6(9), 669-680.

Busso, D. S., Mclaughlin, K, \& Sheridan, M. A. (2017). Dimensions of adversity, physiological reactivity, and externalizing psychopathology in adolescence: Deprivation and threat. Psychosomatic Medicine, 79(2), 162-171.

Casey, B. J., Duhoux, S., \& Malter Cohen, M. (2010). Adolescence: What do transmission, transition, and translation have to do with it? Neuron, 67, 749-760. doi:10.1016/j.neuron.2010.08.033

Chen, Y., Dube, C. M., Rice, C. J., \& Baram, T. Z. (2008). Rapid loss of dendritic spines after stress involves derangement of spine dynamics by corticotropin-releasing hormone. Journal of Neuroscience, 28, 2903-2911. doi:10.1523/jneurosci.0225-08.2008

Ciric, R., Wolf, D. H., Power, J. D., Roalf, D. R., Baum, G. L., Ruparel, K., ... Satterthwaite, T. D. (2017). Benchmarking of participant-level confound regression strategies for the control of motion artifact in studies of functional connectivity. Neuroimage, 154, 174-187. doi:10.1016/j.neuroimage. 2017.03.020

Coe, C. L., \& Shirtcliff, E. A. (2004). Growth trajectory evident at birth affects age of first delivery in female monkeys. Pediatric Research, 55, 914-920. doi:10.1203/01.Pdr.0000125259.45025.4d

Costa Dias, T. G., Iyer, S. P., Carpenter, S. D., Cary, R. P., Wilson, V. B., Mitchell, S. H., Nigg, J. T., \& Fair, D. A. (2015). Characterizing heterogeneity in children with and without ADHD based on reward system connectivity. Developmental Cognitive Neuroscience, 11, 155-174. doi:10.1016/ j.dcn.2014.12.005

D’Andrea, W., Pole, N., DePierro, J., Freed, S., \& Wallace, D. B. (2013). Heterogeneity of defensive responses after exposure to trauma: Blunted autonomic reactivity in response to startling sounds. International Journal of Psychophysiology, 90(1), 80-89.

Danese, A., \& Tan, M. (2014). Childhood maltreatment and obesity: Systematic review and meta-analysis. Molecular Psychiatry, 19, 544-554. doi:10.1038/mp.2013.54

Deoni, S. C., Dean, D. C. 3rd, O’Muircheartaigh, J., Dirks, H., \& Jerskey, B. A. (2012). Investigating white matter development in infancy and early childhood using myelin water faction and relaxation time mapping. Neuroimage, 63, 1038-1053. doi:10.1016/j.neuroimage.2012.07.037

Drury, S. S., Howell, B. R., Jones, C., Esteves, K., Morin, E., Schlesinger, R., ... Sanchez, M. M. (2017). Shaping long-term primate development: Telomere length trajectory as an indicator of early maternal maltreatment and predictor of future physiologic regulation. Developmental Psychopathology, 29, 1539-1551. doi:10.1017/s0954579417001225

Drury, S. S., Sanchez, M. M., \& Gonzalez, A. (2016). When mothering goes awry: Challenges and opportunities for utilizing evidence across rodent, nonhuman primate and human studies to better define the biological consequences of negative early caregiving. Hormones and Behavior, 77, 182192. doi:10.1016/j.yhbeh.2015.10.007

Drury, S., Theall, K., Gleason, M., Smyke, A., De Vivo, I., Wong, J., ... Nelson, C. (2012). Telomere length and early severe social deprivation: Linking early adversity and cellular aging. Molecular Psychiatry, 17(7), 719-727.

Dubois, J., Dehaene-Lambertz, G., Kulikova, S., Poupon, C., Huppi, P. S., \& Hertz-Pannier, L. (2014). The early development of brain white matter: A review of imaging studies in fetuses, newborns and infants. Neuroscience, 276, 48-71. doi:10.1016/j.neuroscience.2013.12.044

Egan, T. M., \& North, R. A. (1985). Acetylcholine acts on m2-muscarinic receptors to excite rat locus coeruleus neurones. British Journal of Pharmacology, 85, 733-735.

Eluvathingal, T. J., Chugani, H. T., Behen, M. E., Juhasz, C., Muzik, O., Maqbool, M., ... Makki, M. (2006). Abnormal brain connectivity in children after early severe socioemotional deprivation: A diffusion tensor imaging study. Pediatrics, 117, 2093-2100. doi:10.1542/peds.2005-1727 
Engberg, G., \& Svensson, T. H. (1980). Pharmacological analysis of a cholinergic receptor mediated regulation of brain norepinephrine neurons. Journal of Neural Transmission, 49, 137-150.

Fair, D. A., Cohen, A. L., Power, J. D., Dosenbach, N. U., Church, J. A., Miezin, F. M., ... Petersen, S. E. (2009). Functional brain networks develop from a "local to distributed" organization. PLoS Computational Biology, 5, e1000381. doi:10.1371/journal.pcbi.1000381

Fair, D. A., Dosenbach, N. U., Church, J. A., Cohen, A. L., Brahmbhatt, S., Miezin, F. M., ... Schlaggar, B. L. (2007). Development of distinct control networks through segregation and integration. Proceedings of the National Academy of Sciences USA, 104, 13507-13512. doi:10.1073/pnas.0705843104

Fair, D. A., Nigg, J. T., Iyer, S., Bathula, D., Mills, K. L., Dosenbach, N. U., ... Milham, M. P. (2012). Distinct neural signatures detected for ADHD subtypes after controlling for micro-movements in resting state functional connectivity MRI data. Frontiers in Systems Neuroscience, 6, 80. doi:10. 3389/fnsys.2012.00080

Fairbanks LA. (1996). Individual differences in maternal styles: Causes and consequences for mothers and offspring. Advances in the Study of Behavior, 579-611.

Fields, R. D. (2008). White matter in learning, cognition and psychiatric disorders. Trends in Neurosciences, 31, 361-370. doi:10.1016/j.tins.2008.04.001

Finkelhor, D., Turner, H. A., Shattuck, A., \& Hamby, S. L. (2013). Violence, crime, and abuse exposure in a national sample of children and youth: An update. JAMA Pediatrics, 167, 614-621. doi:10.1001/jamapediatrics.2013.42

Finlay, J. M., Jedema, H. P., Rabinovic, A. D., Mana, M. J., Zigmond, M. J., \& Sved, A. F. (1997). Impact of corticotropin-releasing hormone on extracellular norepinephrine in prefrontal cortex after chronic cold stress. Journal of Neurochemistry, 69, 144-150.

Foa, E. B., \& Kozak, M. J. (1986). Emotional processing of fear: Exposure to corrective information. Psychological Bulletin, 99, 20-35.

Fonzo, G. A., Simmons, A. N., Thorp, S. R., Norman, S. B., Paulus, M. P., \& Stein, M. B. (2010a). Exaggerated and disconnected insular-amygdalar blood oxygenation level-dependent response to threat-related emotional faces in women with intimate-partner violence posttraumatic stress disorder Biological Psychiatry, 68, 433-441. doi:10.1016/j.biopsych.2010.04.028

Fonzo, G. A., Simmons, A. N., Thorp, S. R., Norman, S. B., Paulus, M. P., \& Stein, M. B. (2010b). Exaggerated and disconnected insular-amygdalar BOLD response to threat-related emotional faces in women with intimatepartner violence PTSD. Biological Psychiatry, 68, 433-441.

Foster, D. J., \& Wilson, M. A. (2006). Reverse replay of behavioural sequences in hippocampal place cells during the awake state. Nature, 440, 680-683. doi:10.1038/nature04587

Franklin, T. B., Russig, H., Weiss, I. C., Graff, J., Linder, N., Michalon, A., .. Mansuy, I. M. (2010). Epigenetic transmission of the impact of early stress across generations. Biological Psychiatry, 68, 408-415. doi:10.1016/ j.biopsych.2010.05.036

Gabard-Durnam, L. J., Flannery, J., Goff, B., Gee, D. G., Humphreys, K. L., Telzer, E., ... Tottenham, N. (2014). The development of human amygdala functional connectivity at rest from 4 to 23 years: A cross-sectional study. Neuroimage, 95, 193-207. doi:10.1016/j.neuroimage.2014.03.038

Gallagher, M., Kapp, B. S., Musty, R. E., \& Driscoll, P. A. (1977). Memory formation: Evidence for a specific neurochemical system in the amygdala. Science, 198, 423-425.

Gee, D. G., Gabard-Durnam, L. J., Flannery, J., Goff, B., Humphreys, K. L., Telzer, E. H., ... Tottenham, N. (2013). Early developmental emergence of human amygdala-prefrontal connectivity after maternal deprivation. Proceedings of the National Academy of Sciences USA, 110, 15638-15643. doi:10.1073/pnas.1307893110

Gee, D. G., Gabard-Durnam, L., Telzer, E. H., Humphreys, K. L., Goff, B., Shapiro, M., ... Tottenham, N. (2014). Maternal buffering of human amygdala-prefrontal circuitry during childhood but not during adolescence. Psychological Science, 25, 2067-2078. doi:10.1177/0956797614550878

Geng, X., Gouttard, S., Sharma, A., Gu, H., Styner, M., Lin, W., ... Gilmore, J. H. (2012). Quantitative tract-based white matter development from birth to age 2years. Neuroimage, 61, 542-557. doi:10.1016/j.neuroimage.2012. 03.057

Goldstein, L. E., Rasmusson, A. M., Bunney, B. S., \& Roth, R. H. (1996). Role of the amygdala in the coordination of behavioral, neuroendocrine, and prefrontal cortical monoamine responses to psychological stress in the rat. Journal of Neuroscience, 16, 4787-4798.

Gorgolewski, K., Burns, C. D., Madison, C., Clark, D., Halchenko, Y. O., Waskom, M. L., \& Ghosh, S. S. (2011). Nipype: A flexible, lightweight and extensible neuroimaging data processing framework in python. Frontiers in Neuroinformatics, 5, 13. doi:10.3389/fninf.2011.00013

Govindan, R. M., Behen, M. E., Helder, E., Makki, M. I., \& Chugani, H. T. (2010). Altered water diffusivity in cortical association tracts in children with early deprivation identified with Tract-Based Spatial Statistics (TBSS). Cerebral Cortex, 20, 561-569. doi:10.1093/cercor/bhp122

Grant, S. J., Aston-Jones, G., \& Redmond, D. E. Jr. (1988). Responses of primate locus coeruleus neurons to simple and complex sensory stimuli. Brain Research Bulletin, 21, 401-410.

Grayson, D. S., Bliss-Moreau, E., Machado, C. J., Bennett, J., Shen, K., Grant, K. A., ... Amaral, D. G. (2016). The rhesus monkey connectome predicts disrupted functional networks resulting from pharmacogenetic inactivation of the amygdala. Neuron, 91, 453-466. doi:10.1016/j.neuron.2016.06.005

Gunnar, M. R., Hostinar, C. E., Sanchez, M. M., Tottenham, N., \& Sullivan, R. M. (2015). Parental buffering of fear and stress neurobiology: Reviewing parallels across rodent, monkey, and human models. Social Neuroscience, 10, 474-478. doi:10.1080/17470919.2015.1070198

Gunnar, M., \& Quevedo, K. (2007). The neurobiology of stress and development. Annual Reviews of Psychology, 58, 145-173. doi:10.1146/ annurev.psych.58.110405.085605

Gunnar, M. R., \& Sullivan, R. M. (2017). The neurodevelopment of social buffering and fear learning: Integration and crosstalk. Social Neuroscience, 12, 1-7. doi:10.1080/17470919.2016.1151824

Hare, T. A., Tottenham, N., Galvan, A., Voss, H. U., Glover, G. H., \& Casey, B. J. (2008). Biological substrates of emotional reactivity and regulation in adolescence during an emotional go-nogo task. Biological Psychiatry, 63, 927934. doi:10.1016/j.biopsych.2008.03.015

Heleniak, C., Mclaughlin, K. A., Ormel, J, \& Riese, H. (2016). Cardiovascular reactivity as a mechanism linking child trauma to adolescent psychopathology. Biological Psychology, 120, 108-119.

Henckens, M. J., van Wingen, G. A., Joels, M., \& Fernandez, G. (2012). Corticosteroid induced decoupling of the amygdala in men. Cerebral Cortex, 22, 2336-2345. doi:10.1093/cercor/bhr313

Hinde, R. A., \& Spencer-Booth, Y. (1967). The behaviour of socially living rhesus monkeys in their first two and a half years. Animal Behavior, 15, 169-196.

Howell, B. R., Ahn, M., Shi, Y., Godfrey, J. R., Hu, X., Zhu, H., ... Sanchez, M. M. (2019). Disentangling the effects of early caregiving experience and heritable factors on brain white matter development in rhesus monkeys. Neuroimage, 197, 625-642. doi:10.1016/j.neuroimage.2019.04.013.

Howell, B. R., Grand, A. P., McCormack, K. M., Shi, Y., LaPrarie, J. L., Maestripieri, D., ... Sanchez, M. M. (2014). Early adverse experience increases emotional reactivity in juvenile rhesus macaques: Relation to amygdala volume. Developmental Psychobiology, 56, 1735-1746. doi:10.1002/dev.21237

Howell, B. R., McCormack, K. M., Grand, A. P., Sawyer, N. T., Zhang, X., Maestripieri, D., ... Sanchez, M. M. (2013). Brain white matter microstructure alterations in adolescent rhesus monkeys exposed to early life stress: Associations with high cortisol during infancy. Biology of Mood and Anxiety Disorders, 3, 21. doi:10.1186/2045-5380-3-21

Howell, B. R., McMurray, M. S., Guzman, D. B., Nair, G., Shi, Y., McCormack, K. M., ... Sanchez, M. M. (2017). Maternal buffering beyond glucocorticoids: Impact of early life stress on corticolimbic circuits that control infant responses to novelty. Social Neuroscience, 12, 50-64. doi:10.1080/17470919. 2016.1200481

Howell, B. R., Neigh, G. N., \& Sánchez, M. M. (2016). Animal models of developmental psychopathology. In Cicchetti, D. (Ed.), Developmental Psychopathology (pp. 166-201). John Wiley \& Sons.

Howell, B. R., \& Sanchez, M. M. (2011). Understanding behavioral effects of early life stress using the reactive scope and allostatic load models. Developmental Psychopathology, 23, 1001-1016. doi:10.1017/ s0954579411000460

Huizinga, D., Haberstick, B. C., Smolen, A., Menard, S., Young, S. E., Corley, R. P., ... Hewitt, J. K. (2006). Childhood maltreatment, subsequent antisocial behavior, and the role of monoamine oxidase A genotype. Biological Psychiatry, 60, 677-683. doi:10.1016/j.biopsych.2005.12.022 
Hutchison, R. M., Hutchison, M., Manning, K. Y., Menon, R. S., \& Everling, S. (2014). Isoflurane induces dose-dependent alterations in the cortical connectivity profiles and dynamic properties of the brain's functional architecture. Human Brain Mapping, 35, 5754-5775. doi:10.1002/ hbm. 22583

Hutchison, R., Womelsdorf, T., Gati, J., Everling, S., \& Menon, R. S. (2013). Resting-state networks show dynamic functional connectivity in awake humans and anesthetized macaques. Human Brain Mapping, 34(9), 2154-2177.

Hutchison, R. M., Womelsdorf, T., Gati, J. S., Leung, L. S., Menon, R. S., \& Everling, S. (2012). Resting-state connectivity identifies distinct functional networks in macaque cingulate cortex. Cerebral Cortex, 22, 1294-1308. doi:10.1093/cercor/bhr181

Iyer, S. P., Shafran, I., Grayson, D., Gates, K., Nigg, J. T., \& Fair, D. A. (2013). Inferring functional connectivity in MRI using Bayesian network structure learning with a modified PC algorithm. Neuroimage, 75, 165-175. doi:10.1016/j.neuroimage.2013.02.054

Jauregui-Huerta, F., Ruvalcaba-Delgadillo, Y., Gonzalez-Castaneda, R., GarciaEstrada, J., Gonzalez-Perez, O., \& Luquin, S. (2010). Responses of glial cells to stress and glucocorticoids. Current Immunology Reviews, 6(3), 195-204.

Johnson, E. O., Kamilaris, T. C., Calogero, A. E., Gold, P. W., \& Chrousos, G. P. (1996). Effects of early parenting on growth and development in a small primate. Pediatric Research, 39, 999-1005. doi:10.1203/00006450-19960600000012

Jones, B. E., \& Yang, T. Z. (1985). The efferent projections from the reticular formation and the locus coeruleus studied by anterograde and retrograde axonal transport in the rat. Journal of Comparative Neurology, 242, 5692. doi:10.1002/cne.902420105

Kaplow, J. B., \& Widom, C. S. (2007). Age of onset of child maltreatment predicts long-term mental health outcomes. Journal of Abnormal Psychology, 116, 176-187. doi:10.1037/0021-843x.116.1.176

Kaufer, D., Friedman, A., Seidman, S., \& Soreq, H. (1998). Acute stress facilitates long-lasting changes in cholinergic gene expression. Nature, 393, 373377. doi: $10.1038 / 30741$

Kelly, A. M., Uddin, L. Q., Biswal, B. B., Castellanos, F. X., \& Milham, M. P. (2008). Competition between functional brain networks mediates behavioral variability. Neuroimage, 39, 527-537. doi:10.1016/j.neuroimage.2007.08.008

Kisiel, C. L., Fehrenbach T., Torgersen, E., Stolbach, B., McClelland, G., Griffin, G., \& Burkman, K. (2014). Constellations of interpersonal trauma and symptoms in child welfare: Implications for a developmental trauma framework. Journal of Family Violence, 29, 1-14.

Koch, H., McCormack, K., Sanchez, M. M., \& Maestripieri, D. (2014). The development of the hypothalamic-pituitary-adrenal axis in rhesus monkeys: Effects of age, sex, and early experience. Developmental Psychobiology, 56, 86-95. doi:10.1002/dev.21093

Kovacs-Balint, Z., Feczko, E., Pincus, M., Earl, E., Miranda-Dominguez, O., Howell, B., ... Sanchez, M. (2018). Early developmental trajectories of functional connectivity along the visual pathways in rhesus monkeys. Cerebral Cortex, 29(8), 3514-3526.

Kumar, A., Behen, M. E., Singsoonsud, P., Veenstra, A. L., Wolfe-Christensen, C., Helder, E., \& Chugani, H. T. (2014). Microstructural abnormalities in language and limbic pathways in orphanage-reared children: A diffusion tensor imaging study. Journal of Child Neurology, 29, 318-325. doi:10.1177/ 0883073812474098

Kumar, S., Cole, R., Chiappelli, F., \& de Vellis, J. (1989). Differential regulation of oligodendrocyte markers by glucocorticoids: Post-transcriptional regulation of both proteolipid protein and myelin basic protein and transcriptional regulation of glycerol phosphate dehydrogenase. Proceedings of the National Academy of Sciences USA, 86, 6807-6811.

Kuwahata, H., Adachi, I., Fujita, K., Tomonaga, M., \& Matsuzawa, T. (2004). Development of schematic face preference in macaque monkeys. Behavioral Processes, 66, 17-21. doi:10.1016/j.beproc.2003.11.002

LeDoux, J. (2007). The amygdala. Current Biology, 17, R868-R874. doi:10.1016/j.cub.2007.08.005

LeDoux, J. E. (2000). Emotion circuits in the brain. Annual Reviews Neuroscience, 23, 155-184. doi:10.1146/annurev.neuro.23.1.155

Lewis, J. W., \& Van Essen, D. C. (2000). Mapping of architectonic subdivisions in the macaque monkey, with emphasis on parieto-occipital cortex. Journal of Comparative Neurology, 428, 79-111.
Li, C. X., Patel, S., Auerbach, E. J., \& Zhang, X. (2013). Dose-dependent effect of isoflurane on regional cerebral blood flow in anesthetized macaque monkeys. Neuroscience Letters, 541, 58-62. doi:10.1016/j.neulet.2013.02.007

Liang, Z., King, J., \& Zhang, N. (2012). Anticorrelated resting-state functional connectivity in awake rat brain. Neuroimage, 59, 1190-1199. doi:10.1016/ j.neuroimage.2011.08.009

Liston, C., Cichon, J. M., Jeanneteau, F., Jia, Z., Chao, M. V., \& Gan, W. B. (2013). Circadian glucocorticoid oscillations promote learning-dependent synapse formation and maintenance. Nature Neuroscience, 16, 698-705. doi:10.1038/nn.3387

Liston, C., \& Gan, W. B. (2011). Glucocorticoids are critical regulators of dendritic spine development and plasticity in vivo. Proceedings of the National Academy of Sciences USA, 108, 16074-16079. doi:10.1073/ pnas. 1110444108

Logothetis, N. K., \& Saleem, K. S. (2012). A Combined MRI and Histology Atlas of the Rhesus Monkey Brain in Stereotaxic Coordinates. San Diego, CA: Academic Press.

Lovallo, W. R., Farag, N. H., Sorocco, K. H., Cohoon, A. J., \& Vincent, A. S. (2012). Lifetime adversity leads to blunted stress axis reactivity: Studies from the Oklahoma Family Health Patterns Project. Biological Psychiatry, 71(4), 344-349.

Lutz, C. K., Lockard, J. S., Gunderson, V. M., \& Grant, K. S. (1998). Infant monkeys' visual responses to drawings of normal and distorted faces. American Journal of Primatology, 44, 169-174. doi:10.1002/(sici) 1098-2345(1998)44:2 < 169::aid-ajp7 > 3.0.co;2-u

Machlin, L., Miller, A. B., Snyder, J, Mclaughlin, K. A., \& Sheridan, M. A. (2019). Differential associations of deprivation and threat with cognitive control and fear conditioning in early childhood. Frontiers in Behavioral Neuroscience, 13, 80. doi:10.3389/fnbeh.2019.00080

Macmillan, H. L., Georgiades, K., Duku, E. K., Shea, A., Steiner, M., Niec, A., ... Schmidt, L. A. (2009). Cortisol response to stress in female youths exposed to childhood maltreatment: Results of the youth mood project. Biological Psychiatry, 66(1), 62-68.

Maestripieri, D. (1998). Parenting styles of abusive mothers in group-living rhesus macaques. Animal Behavior, 55, 1-11.

Maestripieri, D. (1999). The biology of human parenting: Insights from nonhuman primates. Neuroscience \& Biobehavioral Reviews, 23, 411-422.

Maestripieri, D. (2005). Early experience affects the intergenerational transmission of infant abuse in rhesus monkeys. Proceedings of the National Academy of Sciences USA, 102, 9726-9729. doi:10.1073/pnas.0504122102

Maestripieri, D., \& Carroll, K. A. (1998). Risk factors for infant abuse and neglect in group-living rhesus monkeys. Psychological Science, 9, 143-145.

Margulies, D. S., Vincent, J. L., Kelly, C., Lohmann, G., Uddin, L. Q., Biswal, B. B., ... Petrides, M. (2009). Precuneus shares intrinsic functional architecture in humans and monkeys. Proceedings of the National Academy of Sciences USA, 106, 20069-20074. doi:10.1073/pnas.0905314106

Markov, N. T., Misery, P., Falchier, A., Lamy, C., Vezoli, J., Quilodran, R., ... Knoblauch, K. (2011). Weight consistency specifies regularities of macaque cortical networks. Cerebral Cortex, 21, 1254-1272. doi:10.1093/cercor/ bhq201

Matthews, M., \& Fair, D. A. (2015). Research review: Functional brain connectivity and child psychopathology-overview and methodological considerations for investigators new to the field. Journal of Child Psychology and Psychiatry, 56, 400-414. doi:10.1111/jcpp.12335

Mavigner, M., Raper, J., Kovacs-Balint, Z., Gumber, S., O’Neal, J. T., Bhaumik, S. K., ... Chahroudi, A. (2018). Postnatal Zika virus infection is associated with persistent abnormalities in brain structure, function, and behavior in infant macaques. Science Translational Medicine, 10(435), eaao6975. doi:10.1126/scitranslmed.aao6975.

McCormack, K., Howell, B. R., Guzman, D., Villongco, C., Pears, K., Kim, H., ... Sanchez, M. M. (2015). The development of an instrument to measure global dimensions of maternal care in rhesus macaques (Macaca mulatta). American Journal of Primatology, 77, 20-33. doi:10.1002/ajp.22307

McCormack, K., Newman, T. K., Higley, J. D., Maestripieri, D., \& Sanchez, M. M. (2009). Serotonin transporter gene variation, infant abuse, and responsiveness to stress in rhesus macaque mothers and infants. Hormones and Behavior, 55, 538-547. doi:10.1016/j.yhbeh.2009.01.009 
McCormack, K., Sanchez, M. M., Bardi, M., \& Maestripieri, D. (2006). Maternal care patterns and behavioral development of rhesus macaque abused infants in the first 6 months of life. Developmental Psychobiology, 48, 537-550. doi:10.1002/dev.20157

McEwen, B. S. (2004). Protection and damage from acute and chronic stress: Allostasis and allostatic overload and relevance to the pathophysiology of psychiatric disorders. Annals of the New York Academy of Sciences, 1032, 1-7. doi:10.1196/annals.1314.001

McGaugh, J. L. (2002). Memory consolidation and the amygdala: A systems perspective. Trends in Neuroscience, 25, 456.

McLaughlin, K. A., Sheridan, M. A., Gold, A. L., Duys, A., Lambert, H. K., Peverill, M., ... Pine, D. S. (2016). Maltreatment exposure, brain structure, and fear conditioning in children and adolescents. Neuropsychopharmacology, 41, 1956-1964. doi:10.1038/npp.2015.365

Mclaughlin, K., Sheridan, M., Tibu, F., Fox, N., Zeanah, C., \& Nelson, C. (2015). Causal effects of the early caregiving environment on development of stress response systems in children. Proceedings of the National Academy of Sciences, 112, 5637-5642. doi:10.1073/pnas.1423363112

Mcteague, L. M., \& Lang, P. J. (2012). The anxiety spectrum and the reflex physiology of defense: From circumscribed fear to broad distress Depression \& Anxiety, 29(4), 264-281. doi:10.1002/da.21891

Meyer, J., Novak, M., Hamel, A., \& Rosenberg, K. (2014). Extraction and analysis of cortisol from human and monkey hair. Journal of Visualized Experiments, 83, e50882. doi:10.3791/50882

Miranda-Dominguez, O., Mills, B. D., Carpenter, S. D., Grant, K. A., Kroenke, C. D., Nigg, J. T., \& Fair, D. A. (2014a). Connectotyping: Model based fingerprinting of the functional connectome. PLoS One, 9, e111048. doi:10.1371/journal.pone.0111048

Miranda-Dominguez, O., Mills, B. D., Grayson, D., Woodall, A., Grant, K. A., Kroenke, C. D., \& Fair, D. A. (2014b). Bridging the gap between the human and macaque connectome: A quantitative comparison of global interspecies structure-function relationships and network topology. Journal of Neuroscience, 34, 5552-5563. doi:10.1523/jneurosci.4229-13.2014

Moriceau, S., \& Sullivan, R. M. (2006). Maternal presence serves as a switch between learning fear and attraction in infancy. Nature Neuroscience, 9, 1004-1006. doi:10.1038/nn1733

Morin, E. L., Howell, B. R., Meyer, J. S., \& Sanchez, M. M. (2019). Effects of early maternal care on adolescent attention bias to threat in nonhuman primates. Developmental Cognitive Neuroscience, 38, 100643. https://doi.org/ 10.1016/j.dcn.2019.100643.

Morin, E. L., Howell, B. R., Reding, K., Guzman, D. B., Feczko, E., Earl, E., ... Shi, Y. (2015). Early Maternal Care Modulates the Development of Emotional Neurocircuitry in Nonhuman Primates: Amygdala Functional Connectivity. 45th Annual Meeting of the Society for Neuroscience (SfN).

Morin, E. L., Wakeford, A. G. P., Howell, B. R., Guzman, D. B., Siebert, E., Kazama, A. M., ... Sanchez, M. (2018). Maternal care controls the development of fear learning in adolescent nonhuman primates: relationship with prefrontal 5htla receptor binding. Paper presented at the 48th Annual Meeting of the Society for Neuroscience (SfN), San Diego, CA.

Morris, J. S., Ohman, A., \& Dolan, R. J. (1998). Conscious and unconscious emotional learning in the human amygdala. Nature, 393, 467-470 doi:10.1038/30976

Murphy, K, \& Fox, M. (2017). Towards a consensus regarding global signal regression for resting state functional connectivity MRI. Neuroimage, 154, 169-173.

Muschinski, J., Feczko, E., Brooks, J. M., Collantes, M., Heitz, T. R., \& Parr, L. A. (2016). The development of visual preferences for direct versus averted gaze faces in infant macaques (Macaca mulatta). Developmental Psychobiology, 58, 926-936. doi:10.1002/dev.21421

Nalci, A., Rao, B. D., \& Liu, T. T. (2017). Global signal regression acts as a temporal downweighting process in resting-state fMRI. Neuroimage, 152, 602618. doi:10.1016/j.neuroimage.2017.01.015

Ouellet-Morin, I, Odgers, C. L., Danese, A., Bowes, L., Shakoor, S., Papadopoulos, A, ... Arseneault, L. (2011). Blunted cortisol responses to stress signal social and behavioral problems among maltreated/bullied 12year-old children. Biological Psychiatry, 70(11), 1015-1023.

Palanca, B. J., Mitra, A., Larson-Prior, L., Snyder, A. Z., Avidan, M. S., \& Raichle, M. E. (2015). Resting-state functional magnetic resonance imaging correlates of sevoflurane-induced unconsciousness. Anesthesiology, 123, 346-356. doi:10.1097/aln.0000000000000731

Parr, L. A., Murphy, L., Feczko, E., Brooks, J., Collantes, M., \& Heitz, T. R. (2016). Experience-dependent changes in the development of face preferences in infant rhesus monkeys. Developmental Psychobiology, 58, 1002 1018. doi:10.1002/dev.21434

Paxinos G, Huang X.-F., \& Toga, A. W. (2000). The Rhesus Monkey Brain in Sterotaxic Coordinates. San Diego, CA: Academic Press.

Petrullo, L. A., Mandalaywala, T. M., Parker, K. J., Maestripieri, D., \& Higham, J. P. (2016). Effects of early life adversity on cortisol/salivary alpha-amylase symmetry in free-ranging juvenile rhesus macaques. Hormones and Behavior, 86, 78-84. doi:10.1016/j.yhbeh.2016.05.004

Power, J. D., Barnes, K. A., Snyder, A. Z., Schlaggar, B. L., \& Petersen, S. E. (2012). Spurious but systematic correlations in functional connectivity MRI networks arise from subject motion. Neuroimage, 59, 2142-2154. doi:10.1016/j.neuroimage.2011.10.018

Power, J. D., Laumann, T. O., Plitt, M., Martin, A., \& Petersen, S. E. (2017). On global fMRI signals and simulations. Trends in Cognitive Sciences, 21, 911913. doi:10.1016/j.tics.2017.09.002

Power, J. D., Mitra, A., Laumann, T. O., Snyder, A. Z., Schlaggar, B. L., \& Petersen, S. E. (2014). Methods to detect, characterize, and remove motion artifact in resting state fMRI. Neuroimage, 84, 320-341. doi:10.1016/ j.neuroimage.2013.08.048

Qin, S., Young, C. B., Supekar, K., Uddin, L. Q., \& Menon, V. (2012). Immature integration and segregation of emotion-related brain circuitry in young children. Proceedings of the National Academy of Sciences USA, 109, 7941-7946. doi:10.1073/pnas.1120408109

Rasmussen, K., Morilak, D. A., \& Jacobs, B. L. (1986). Single unit activity of locus coeruleus neurons in the freely moving cat. I. During naturalistic behaviors and in response to simple and complex stimuli. Brain Research, 371, 324-334.

Reding, K. M., Grayson, D. S., Miranda-Dominguez, O., Ray, S., Wilson, M. E., Toufexis, D., ... Sanchez, M. M. (2019). Effects of social subordination and estradiol on resting-state amygdala functional connectivity in adult female rhesus monkeys. Journal of Neuroendocrinology, e12822. doi:10.1111/jne.12822.

Reding, K. M., Grayson, D. S., Miranda-Dominguez, O., Ray, S., Wilson, M. E., Toufexis, D., ... Sanchez, M. M. (2020). Effects of social subordination and oestradiol on resting-state amygdala functional connectivity in adult female rhesus monkeys. Journal of Neuroendocrinology, 32, e12822. doi:10.1111/ jne. 12822

Rockstroh, B., \& Elbert, T. (2010). Traces of fear in the neural web-magnetoencephalographic responding to arousing pictorial stimuli. International Journal of Psychophysiology, 78, 14-19. doi:10.1016/j.ijpsycho.2010.01.012

Roozendaal, B., McEwen, B. S., \& Chattarji, S. (2009). Stress, memory and the amygdala. Nature Reviews Neuroscience, 10, 423-433. doi:10.1038/nrn2651

Roy, A. K., Shehzad, Z., Margulies, D. S., Kelly, A. M., Uddin, L. Q., Gotimer, K., .. Milham, M. P. (2009). Functional connectivity of the human amygdala using resting state fMRI. Neuroimage, 45, 614-626. doi:10.1016/ j.neuroimage.2008.11.030

Russchen, F. T., Bakst, I., Amaral, D. G., \& Price, J. L. (1985). The amygdalostriatal projections in the monkey. An anterograde tracing study. Brain Research, 329, 241-257.

Rutter, M., Andersen-Wood, L., Beckett, C., Bredenkamp, D., Castle, J., Groothues, C., ... O'Connor, T. G. (1999). Quasi-autistic patterns following severe early global privation. English and Romanian Adoptees (ERA) Study Team. Journal of Child Psychology and Psychiatry, 40, 537-549.

Sallet, J., Mars, R. B., Noonan, M. P., Andersson, J. L., O’Reilly, J. X., Jbabdi, S., ... Rushworth, M. F. (2011). Social network size affects neural circuits in macaques. Science, 334, 697-700. doi:10.1126/science.1210027

Sanchez, M. M. (2006). The impact of early adverse care on HPA axis development: Nonhuman primate models. Hormones and Behavior, 50, 623-631. doi:10.1016/j.yhbeh.2006.06.012

Sanchez, M. M., Alagbe, O., Felger, J. C., Zhang, J., Graff, A. E., Grand, A. P., ... Miller, A. H. (2007). Activated p38 MAPK is associated with decreased CSF 5-HIAA and increased maternal rejection during infancy in rhesus monkeys. Molecular Psychiatry, 12, 895-897. doi:10.1038/sj.mp.4002025

Sanchez, M. M., Hearn, E. F., Do, D., Rilling, J. K., \& Herndon, J. G. (1998). Differential rearing affects corpus callosum size and cognitive function of rhesus monkeys. Brain Research, 812, 38-49. 
Sanchez, M. M., Ladd, C. O., \& Plotsky, P. M. (2001). Early adverse experience as a developmental risk factor for later psychopathology: Evidence from rodent and primate models. Developmental Psychopathology, 13, 419-449.

Sanchez, M. M., McCormack, K., Grand, A. P., Fulks, R., Graff, A., \& Maestripieri, D. (2010). Effects of sex and early maternal abuse on adrenocorticotropin hormone and cortisol responses to the corticotropinreleasing hormone challenge during the first 3 years of life in group-living rhesus monkeys. Developmental Psychopathology, 22, 45-53. doi:10. 1017/s0954579409990253

Sanchez, M. M., McCormack, K. M., \& Howell, B. R. (2015). Social buffering of stress responses in nonhuman primates: Maternal regulation of the development of emotional regulatory brain circuits. Social Neuroscience, 10, 512526. doi:10.1080/17470919.2015.1087426

Sanchez, M. D., Milanes, M. V., Pazos, A., Diaz, A., \& Laorden, M. L. (2000a). Autoradiographic evidence of delta-opioid receptor downregulation after prenatal stress in offspring rat brain. Pharmacology, 60, 13-18. doi:10. $1159 / 000028341$

Sanchez, M. M., Young, L. J., Plotsky, P. M., \& Insel, T. R. (2000b). Distribution of corticosteroid receptors in the rhesus brain: Relative absence of glucocorticoid receptors in the hippocampal formation. Journal of Neuroscience, 20, 4657-4668.

Schmahmann J. D., Pandya D. N. (2006). Fiber pathways of the brain. Oxford: Oxford University Press.

Shi, Y., Budin, F., Yapuncich, E., Rumple, A., Young, J., Payne, C., ... Styner, M. A. (2017). UNC-Emory infant atlases for macaque brain image analysis: Postnatal brain development through 12 months. Frontiers in Neuroscience, 10, 617.

Smith, S. M., Jenkinson, M., Woolrich, M. W., Beckmann, C. F., Behrens, T. E., Johansen-Berg, H., ... Matthews, P. M. (2004). Advances in functional and structural MR image analysis and implementation as FSL. Neuroimage, 23, S208-219. doi:10.1016/j.neuroimage.2004.07.051

Spinazzola, J., Hodgdon, H., Liang, L., Ford, J. D., Layne, C. M., Pynoos, R., ... Kisiel, C. (2014). Unseen wounds: The contribution of psychological maltreatment to child and adolescent mental health and risk outcomes. Psychological Trauma: Theory, Research, Practice, and Policy, 6, S18-S28.

Steinberg, A., Pynoos, R., Briggs, E. C., Gerrity, E. T., Layne, C. M., Vivrette, R. L., ... Fairbank, J. A. (2014). The national child traumatic stress network core data set: Emerging findings, future directions, and implications for theory, research, practice, and policy. Psychological Trauma: Theory, Research, Practice, and Policy, 6, S50-S57.

Styner, M., Knickmeyer, R., Joshi, S., Coe, C., Short, S. J., \& Gilmore, J.. (2007). Automatic brain segmentation in rhesus monkeys. P Soc Photo-Opt Ins, 6512, L5122.

Sugita, Y. (2008). Face perception in monkeys reared with no exposure to faces. Proceedings of the National Academy of Sciences USA, 105, 394-398. doi:10.1073/pnas.0706079105

Sullivan, R. M., Landers, M., Yeaman, B., \& Wilson, D. A. (2000). Good memories of bad events in infancy. Nature, 407, 38-39. doi:10.1038/35024156

Tang, C. Y., \& Ramani, R. (2016). fMRI and anesthesia. International Anesthesiology Clinics, 54(1), 129-142. doi:10.1097/AIA.0000000000000081

Tarullo, A. R., \& Gunnar, M. R. (2006). Child maltreatment and the developing HPA axis. Hormones and Behavior, 50, 632-639. doi:10.1016/ j.yhbeh.2006.06.010

Teicher, M. H., Andersen, S. L., Polcari, A., Anderson, C. M., Navalta, C. P., \& Kim, D. M. (2003). The neurobiological consequences of early stress and childhood maltreatment. Neuroscience \& Biobehavioral Reviews, 27, 33-44.

Teicher, M. H., Samson, J. A., Anderson, C. M., \& Ohashi, K. (2016). The effects of childhood maltreatment on brain structure, function and connectivity. Nature Reviews Neuroscience, 17, 652-666. doi:10.1038/ nrn.2016.111

Thomason, M. E., Marusak, H. A., Tocco, M. A., Vila, A. M., McGarragle, O., \& Rosenberg, D. R. (2015). Altered amygdala connectivity in urban youth exposed to trauma. Social Cognitive and Affective Neuroscience, 10, 14601468. doi:10.1093/scan/nsv030

Thomason, M. E., \& Thompson, P. M. (2011). Diffusion imaging, white matter, and psychopathology. Annual Reviews of Clinical Psychology, 7, 63-85. doi:10.1146/annurev-clinpsy-032210-104507

Tottenham, N. (2015). Social scaffolding of human amygdala-mPFCcircuit development. Social Neuroscience, 10, 489-499. doi:10.1080/ 17470919.2015.1087424

Trickett, P. K., Gordis, E., Peckins, M. K., \& Susman, E. J. (2014). Stress reactivity in maltreated and comparison male and female young adolescents. Child Maltreatment, 19(1), 27-37.

Troisi, A., \& D’Amato, F. R. (1984). Ambivalence in monkey mothering. Infant abuse combined with maternal possessiveness. The Journal or Nervous and Mental Disease, 172, 105-108.

Van Bockstaele, E. J., Bajic, D., Proudfit, H., \& Valentino, R. J. (2001). Topographic architecture of stress-related pathways targeting the noradrenergic locus coeruleus. Physiology \& Behavior, 73, 273-283.

VanTieghem, M. R., \& Tottenham, N. (2018). Neurobiological programming of early life stress: Functional development of amygdala-prefrontal circuitry and vulnerability for stress-related psychopathology. Current Topics in Behavioral Neurosciences, 38, 117-136. doi:10.1007/7854_2016_42

Vincent, J. L., Patel, G. H., Fox, M. D., Snyder, A. Z., Baker, J. T., Van Essen, D. C., ... Raichle, M. E. (2007). Intrinsic functional architecture in the anaesthetized monkey brain. Nature, 447, 83-86. doi:10.1038/nature05758

Vohr, B. R., Wright, L. L., Dusick, A. M., Mele, L., Verter, J., Steichen, J. J., ... Kaplan, M. D. (2000). Neurodevelopmental and functional outcomes of extremely low birth weight infants in the National Institute of Child Health and Human Development Neonatal Research Network, 1993-1994. Pediatrics, 105, 1216-1226.

Vyas, A., Mitra, R., Shankaranarayana Rao, B. S., \& Chattarji, S. (2002). Chronic stress induces contrasting patterns of dendritic remodeling in hippocampal and amygdaloid neurons. Journal of Neuroscience, 22, 6810-6818. doi:20026655.

Weber, D. L. (2008). Information processing bias in post-traumatic stress disorder. Open Neuroimaging Journal, 2, 29-51. doi:10.2174/1874440000802010029

Webster, M. J., Ungerleider, L. G., \& Bachevalier, J. (1991a). Connections of inferior temporal areas TE and TEO with medial temporal-lobe structures in infant and adult monkeys. Journal of Neuroscience, 11, 1095-1116.

Webster, M. J., Ungerleider, L. G., \& Bachevalier, J. (1991b). Lesions of inferior temporal area TE in infant monkeys alter cortico-amygdalar projections. Neuroreport, 2, 769-772.

Woolrich, M. W., Jbabdi, S., Patenaude, B., Chappell, M., Makni, S., Behrens, T., ... Smith, S. M. (2009). Bayesian analysis of neuroimaging data in FSL. Neuroimage, 45, S173-186. doi:10.1016/j.neuroimage.2008.10.055

Yan, C. G., Cheung, B., Kelly, C., Colcombe, S., Craddock, R. C., Di Martino, A., ... Milham, M. P. (2013). A comprehensive assessment of regional variation in the impact of head micromovements on functional connectomics. Neuroimage, 76, 183-201. doi:10.1016/j.neuroimage.2013.03.004

Zatorre, R. J., Fields, R. D., \& Johansen-Berg, H. (2012). Plasticity in gray and white: Neuroimaging changes in brain structure during learning. Nature Neuroscience, 15, 528-536. doi:10.1038/nn.3045

Zhang, W., Jiang, X., Zhang, S., Howell, B. R., Zhao, Y., Zhang, T., ... Liu, T. (2017). Connectome-scale functional intrinsic connectivity networks in macaques. Neuroscience, 364, 1-14. doi:10.1016/j.neuroscience.2017.08.022 